Pacific

Journal of

Mathematics

\title{
ON J-HOLOMORPHIC CURVES \\ IN ALMOST COMPLEX MANIFOLDS WITH ASYMPTOTICALLY CYLINDRICAL ENDS
}

ERKAO BAO 


\title{
ON $J$-HOLOMORPHIC CURVES IN ALMOST COMPLEX MANIFOLDS WITH ASYMPTOTICALLY CYLINDRICAL ENDS
}

\author{
ERKAO BAO
}

\begin{abstract}
Symplectic field theory is the study of $J$-holomorphic curves in almost complex manifolds with cylindrical ends. One natural generalization is to replace "cylindrical" by "asymptotically cylindrical". We generalize a number of asymptotic results about the behavior of $\boldsymbol{J}$-holomorphic curves near infinity to the asymptotically cylindrical setting. We also sketch how these asymptotic results allow compactness theorems in symplectic field theory to be extended to the asymptotically cylindrical case.
\end{abstract}

1. Introduction

2. Asymptotically cylindrical almost complex structures 292

3. Proof of main results 296

4. Almost complex manifolds with asymptotically cylindrical ends 318

Acknowledgements $\quad 323$

References

\section{Introduction}

Introduced by Gromov [1985], J-holomorphic curves have been studied intensively in closed symplectic manifolds. Hofer [1993] studied the behaviors of $J$-holomorphic curves in symplectizations of contact manifolds, which are noncompact. Shortly after that, Eliashberg, Givental and Hofer [2000] invented symplectic field theory, which greatly helps us understand symplectic manifolds and contact manifolds. In most of the previous literature, the almost complex structure $J$ is cylindrical near the ends of the noncompact symplectic manifolds. Here cylindrical means that $J$ is independent of the radial direction. In [Bourgeois et al. 2003] the notion was introduced of an asymptotically cylindrical almost complex structure, which is a natural generalization of a cylindrical almost complex structure. However, no results corresponding to the notion of asymptotically cylindrical almost

MSC2010: primary 53D05, 53D10, 53D12, 53D40, 53D42; secondary 58J05.

Keywords: asymptotically cylindrical almost complex structure, symplectic field theory, compactness,

Hofer energy, J-holomorphic curve, Morse-Bott, stable Hamiltonian structure. 
complex structures in that paper have been proven. Intuitively, we expect similar results as in the cylindrical case. However, the original proofs rely heavily on the cylindrical nature of the almost complex structure, which prevents us from a direct generalization to the asymptotically cylindrical case. In this paper, we give a modified definition of asymptotically cylindrical almost complex structure, which includes an exponential decay condition that is satisfied in all interesting examples, and prove some parallel analytical results as in the cylindrical case. Based on these results we can compactify the moduli space of $J$-holomorphic curves in almost complex manifolds with asymptotically cylindrical ends by adding the holomorphic buildings introduced by [Bourgeois et al. 2003].

This generalization is needed for application purposes, since in many cases the natural almost complex structure is only asymptotically cylindrical (see Examples 2.5 and 4.1). For instance, we can use the generalized results to prove Gromov's monotonicity theorem with multiplicity (see [Bao 2014]). We also take this chance to fill in some gaps in the literature.

In the asymptotically cylindrical case, the proofs of some theorems are significantly different and more sophisticated than the proofs in the cylindrical case (see the proofs of Proposition 3.4, Theorem 2.8 and Theorem 3.7, for example). The extra difficulties mainly come from the following two facts: (1) the translations in the cylindrical almost complex manifold are not $J$-holomorphic anymore; (2) the unmodified Hofer energy is not positive when restricted to $J$ complex planes, and the modified Hofer energy is not closed. Crucial uses of Gromov's monotonicity theorem are the main ingredients to overcoming these extra difficulties.

In Section 2, we give the definition of asymptotically cylindrical almost complex manifolds and the definition of Hofer energy of $J$-holomorphic curves in this context.

In Section 3, we give the proofs of the main results listed in Section 2. The proofs follow the schemes of [Hofer 1993; Hofer et al. 2001; Hofer et al. 2002; Bourgeois 2002; Bourgeois et al. 2003].

In Section 4, we give the definition of almost complex manifolds with asymptotically cylindrical ends and the definition of Hofer energy in this context. Finally we state and outline the proof of the compactness result in this context.

\section{Asymptotically cylindrical almost complex structures}

2A. Definitions. Let $V$ be a smooth closed oriented manifold of dimension $2 n+1$, and let $J$ be a smooth almost complex structure in $W:=\mathbb{R}^{+} \times V$. Assume that the orientation of $W$ determined by $J$ is the same as the orientation coming from the standard orientation of $\mathbb{R}^{+}$and the orientation of $V$. Let $\boldsymbol{R}:=J(\partial / \partial r)$ be a smooth vector field on $W$, and let $\xi$ be a subbundle of the tangent bundle $T W$ 
defined by $\xi_{(r, v)}=\left(0 \times T_{v} V\right) \cap J\left(0 \times T_{v} V\right) \subset T_{(r, v)} W$, for $(r, v) \in W$. The tangent bundle $T W$ splits as $T W=\mathbb{R}(\partial / \partial r) \oplus \mathbb{R}(\boldsymbol{R}) \oplus \xi$.

Define a 1-form $\lambda$ on $W$ by $\lambda(\xi)=0, \lambda(\partial / \partial r)=0, \lambda(\boldsymbol{R})=1$, and a 1-form $\sigma$ on $W$ by $\sigma(\xi)=0, \sigma(\partial / \partial r)=1, \sigma(\boldsymbol{R})=0$.

We call a tensor on $W$ translationally invariant if it is independent of the $r$-coordinate. Let $f_{s}: W \rightarrow W$ be the translation along the $\mathbb{R}^{+}$-direction defined by $f_{s}(r, v):=(r+s, v)$.

Definition 2.1. Under the above notation, $J$ is called asymptotically cylindrical at positive infinity if, for all $l \in \mathbb{Z}_{\geq 0}$, the following five conditions are satisfied:

(AC1) There exists a smooth translationally invariant almost complex structure $J_{\infty}$ on $W$ and constants $K_{l}^{+}, \delta_{l}>0$ such that

$$
\left\|\left.\nabla^{l}\left(J-J_{\infty}\right)\right|_{[r,+\infty) \times V}\right\|_{C^{0}} \leq K_{l}^{+} e^{-\delta_{l} r}
$$

for all $r \geq 0$, where $\|\cdot\|_{C^{0}}$ is computed using a translationally invariant metric $g_{W}$ on $W$ (for example, $g_{W}=d r^{2}+g_{V}$ ), and $\nabla$ is the corresponding Levi-Civita connection. We further require that $K_{l}^{+}$is sufficiently small such that the $\omega$ defined in Equation (2) satisfies requirements (a) and (b) in Section 2B. (Remark 2.2 explains that $K_{l}^{+}$being small is not restrictive.)

(AC2) $i\left(\boldsymbol{R}_{\infty}\right) d \lambda_{\infty}=0$, where $\boldsymbol{R}_{\infty}:=\lim _{s \rightarrow \infty} f_{s}^{*} \boldsymbol{R}, \lambda_{\infty}:=\lim _{s \rightarrow \infty} f_{s}^{*} \lambda$, and both limits exist by (AC1).

(AC3) $\boldsymbol{R}_{\infty}(r, v)=J_{\infty}(\partial / \partial r) \in 0 \times T_{v} V$.

(AC4) There exists a closed 2-form $\omega_{\infty}$ on $V$ such that $i\left(\boldsymbol{R}_{\infty}\right) \omega_{\infty}=0$.

(AC5) $\omega_{\infty}\left(\cdot, J_{\infty} \cdot\right)$ is a metric on $\xi_{\infty}$, where $\xi_{\infty}=\lim _{s \rightarrow \infty} f_{s}^{*} \xi$.

Remark 2.2. The definition we use is slightly different from the one in [Bourgeois et al. 2003]. We require that $J$ converges to $J_{\infty}$ exponentially fast in condition (AC1). This is the accurate condition to guarantee that the $J$-holomorphic curve converges to the periodic orbits of $\boldsymbol{R}_{\infty}$ exponentially fast by the footnote of formula (35). If we are only interested in the behavior of a $J$-holomorphic curve near infinity, then the requirement that $K_{l}^{+}$is small can be achieved by restricting $W$ to $r \geq r_{0}$ for some large $r_{0}$.

We can restate the above conditions using the notion of hamiltonian structure as in [Eliashberg 2007]. That the 2-form $\omega_{\infty}$ has rank $2 n$ says that $\left(V, \omega_{\infty}\right)$ is a hamiltonian structure. The conditions (AC3), $i\left(\boldsymbol{R}_{\infty}\right) \omega_{\infty}=0=i\left(\boldsymbol{R}_{\infty}\right) d \lambda_{\infty}$ and $\lambda_{\infty}\left(\boldsymbol{R}_{\infty}\right)=1$ say that $\left(V, \omega_{\infty}\right)$ is a stable hamiltonian structure. The condition $\xi_{\infty}=\operatorname{ker} \lambda_{\infty}$, that $J_{\infty}$ is an almost complex structure on $\xi_{\infty}$, and that $J_{\infty}$ is compatible with $\omega_{\infty}$ (by (AC5)) imply that $\left(\lambda_{\infty}, J_{\infty}\right)$ is a framing of $\left(V, \omega_{\infty}\right)$. If in addition $\omega_{\infty}=d \lambda_{\infty}$, then we say $\left(V, \omega_{\infty}\right)$ is of contact type. 
We call $(\lambda, J)$ defined as above an asymptotically cylindrical framing of the stable hamiltonian structure $\left(V, \omega_{\infty}\right)$.

Similarly, we can define the notion of $J$ being asymptotically cylindrical on $\mathbb{R}^{-} \times V$ at $-\infty$. When we say $J$ is asymptotically cylindrical, we choose $\omega_{ \pm \infty}$ without mention.

The following definition is the case considered in [Hofer 1993; Hofer et al. 2001; Hofer et al. 2002; Bourgeois 2002; Bourgeois et al. 2003].

Definition 2.3. An almost complex structure $J$ on $\mathbb{R}^{ \pm} \times V$ is said to be a cylindrical almost complex structure at $\pm \infty$ if $J$ is an asymptotically cylindrical almost complex structure at $\pm \infty$ and $J$ is translationally invariant near $\pm \infty$.

An almost complex structure $J$ on $\mathbb{R} \times V$ is said to be a cylindrical almost complex structure if $J$ is asymptotically cylindrical at both $\infty$ and $-\infty$ and $J$ is translationally invariant.

Example 2.4 (Symplectization). Assume $(V, \xi)$ is a contact manifold with contact 1 -form $\lambda$ and Reeb vector field $\boldsymbol{R}$, i.e., $\xi=\operatorname{ker} \lambda, \lambda \wedge(d \lambda)^{n} \neq 0, i_{\boldsymbol{R}} d \lambda=0$, and $\lambda(\boldsymbol{R})=1$. Let $\omega_{\infty}=d \lambda$ and let $J_{\xi}$ be an almost complex structure in $\xi$ such that it is compatible with $\left.\omega_{\infty}\right|_{\xi}$, i.e., $d \lambda\left(\cdot, J_{\xi} \cdot\right)$ is a metric on $\xi$. We extend $J_{\xi}$ to $\mathbb{R} \times V$ by setting $J(\partial / \partial r)=\boldsymbol{R}$. Then $J$ is a cylindrical almost complex structure and, in particular, an asymptotically cylindrical almost complex structure at $\pm \infty$.

Refer to [Bourgeois et al. 2003] for other interesting examples of cylindrical almost complex structures.

Example 2.5. Assume $J$ is a smooth almost complex structure on $\mathbb{R}^{2 n+2}$ with $J(0)=J_{0}(0)$, where $J_{0}$ is the standard complex structure on $\mathbb{R}^{2 n+2}$. Consider $\mathbb{R}^{2 n+2} \backslash\{0\}$ and pick a polar coordinate chart

$$
\varphi: \mathbb{R}^{-} \times S^{2 n+1} \rightarrow \mathbb{R}^{2 n+2} \backslash\{0\}, \quad(r, \Theta) \mapsto e^{r} \Theta,
$$

where we view $S^{2 n+1}$ as the unit sphere inside $\mathbb{R}^{2 n+2}$. Let $\lambda_{-\infty}$ be the standard contact form on $S^{2 n+1}$. Define the 2 -form $\omega_{-\infty}$ on $\mathbb{R}^{-} \times S^{2 n+1}$ by $\omega_{-\infty}=d \lambda_{-\infty}$. Now it is clear that $\left.J\right|_{\mathbb{R}^{-} \times S^{2 n+1}}$ is an asymptotically cylindrical almost complex structure near $-\infty$.

By (AC1) and (AC3) we can see that $\boldsymbol{R}_{\infty}$ is a translationally invariant vector field on $W$ and that it is tangent to each level set $\{r\} \times V$, so we can view $\boldsymbol{R}_{\infty}$ as a vector field on $V$. Let $\phi^{t}$ be the flow of $\boldsymbol{R}_{\infty}$ on $V$, i.e., let $\phi^{t}: V \rightarrow V$ satisfy $(d / d t) \phi^{t}=\boldsymbol{R}_{\infty} \circ \phi^{t}$. Then we have

$$
\frac{d}{d t}\left[\left(\phi^{t}\right)^{*} \lambda_{\infty}\right]=\left(\phi^{t}\right)^{*}\left(i\left(\boldsymbol{R}_{\infty}\right) d \lambda_{\infty}+\operatorname{di}\left(\boldsymbol{R}_{\infty}\right) \lambda_{\infty}\right)=0 .
$$

Hence $\phi^{t}$ preserves $\lambda_{\infty}$ and thus also $\xi_{\infty}$. Similarly, $\phi^{t}$ preserves $\omega_{\infty}$. 
Let's denote by $\mathcal{P}$ the set of periodic trajectories, counting their multiples, of the vector field $\boldsymbol{R}_{\infty}$ restricting to $V$. Notice that any smooth family of periodic trajectories from $\mathcal{P}$ has the same period by Stokes' theorem.

Definition 2.6. A $T$-periodic orbit $\gamma$ of $\boldsymbol{R}_{\infty}$ is called nondegenerate if $\left.d \phi^{T}\right|_{\xi_{\infty}(\gamma(0))}$ does not have 1 as an eigenvalue, where $\phi^{t}$ is the flow of $\boldsymbol{R}_{\infty}$. We say that $J$ is nondegenerate if all the periodic solutions of $\boldsymbol{R}_{\infty}$ are nondegenerate.

A weaker requirement for $J$ than nondegenerate is Morse-Bott.

Definition 2.7. We say that $J$ is of the Morse-Bott type if, for every $T>0$, the subset $N_{T} \subset V$ formed by the closed trajectories from $\mathcal{P}$ of period $T$ is a smooth closed submanifold of $V$ such that the rank of $\left.\omega_{\infty}\right|_{N_{T}}$ is locally constant and $T_{p} N_{T}=\operatorname{ker}\left(d \phi^{T}-\mathrm{Id}\right)_{p}$.

We always assume $J$ is of Morse-Bott type in this paper.

2B. Energy of J-holomorphic curves. Let $J$ be an asymptotically cylindrical almost complex structure on $W:=\mathbb{R}^{+} \times V$. Let's denote the projections from $T W=\mathbb{R}(\partial / \partial r) \oplus \mathbb{R}(\boldsymbol{R}) \oplus \xi$ to each subbundle by $\pi_{r}, \pi_{\boldsymbol{R}}$ and $\pi_{\xi}$. It is convenient to introduce a 2 -form $\omega$ on $W$ by

$$
\omega(x, y)=\frac{1}{2}\left[\omega_{\infty}\left(\pi_{\xi} x, \pi_{\xi} y\right)+\omega_{\infty}\left(J \pi_{\xi} x, J \pi_{\xi} y\right)\right] .
$$

It is easy to check that $i(\partial / \partial r) \omega=0=i(\boldsymbol{R}) \omega$. We assume that $K_{l}^{+}$in (AC1) is sufficiently small for all $l \in \mathbb{Z}_{\geq 0}$ such that $\omega$ satisfies the following two conditions:

(a) $\left.\omega\right|_{\xi}(\cdot, J \cdot)$ is a metric on $\xi$.

(b) There exist constants $\varepsilon_{l}, \delta_{l}>0$ such that, for all $r \geq 0$,

$$
\left\|\left.\left(\omega-\omega_{\infty}\right)\right|_{[r,+\infty) \times V}\right\|_{C^{l}} \leq \varepsilon_{l} e^{-\delta_{l} r} .
$$

Let $(\Sigma, j)$ be a punctured Riemann surface (with or without boundary) and let $\tilde{u}=(a, u):(\Sigma, j) \rightarrow(W, J)$ be a $J$-holomorphic curve, i.e., $T \tilde{u} \circ j=J(\tilde{u}) \circ T \tilde{u}$. The following definition is a modification of Hofer energy in the cylindrical almost complex structure case. The $\omega$-energy and $\lambda$-energy are defined, respectively, as

$$
E_{\omega}(\tilde{u})=\int_{\Sigma} \tilde{u}^{*} \omega, \quad E_{\lambda}(\tilde{u})=\sup _{\phi \in \mathcal{C}} \int_{\Sigma} \tilde{u}^{*}(\phi(r) \sigma \wedge \lambda),
$$

where $\mathcal{C}=\left\{\phi \in C_{c}^{\infty}(\mathbb{R},[0,1]): \int_{-\infty}^{+\infty} \phi(x) d x=1\right\}^{1}$, and $\lambda, \sigma$ are defined as in the beginning of Section 2A. Let's define the energy of $\tilde{u}$ by

$$
E(\tilde{u})=E_{\omega}(\tilde{u})+E_{\lambda}(\tilde{u}) .
$$

${ }^{1}$ In [Bourgeois et al. 2003], the set $\mathcal{C}$ is given by $\mathcal{C}=\left\{\phi \in C_{c}^{\infty}\left(\mathbb{R}, \mathbb{R}^{+}\right): \int_{-\infty}^{+\infty} \phi(x) d x=1\right\}$. It is easier to get uniform energy bounds using the modified definition in the case when the almost complex structure is only asymptotically cylindrical. 
Equip $\mathbb{R}^{+} \times S^{1}$ with the standard complex structure and coordinate $(s, t)$, and consider a $J$-holomorphic map $\tilde{u}=(a, u): \mathbb{R}^{+} \times S^{1} \rightarrow W$. Here we view $S^{1}$ as $\mathbb{R} / \mathbb{Z}$. Notice that

$$
\begin{gathered}
\tilde{u}^{*} \omega=\omega\left(\pi_{\xi} \tilde{u}_{s}, J(\tilde{u}) \pi_{\xi} \tilde{u}_{s}\right) d s \wedge d t, \\
\tilde{u}^{*}(\phi(r) \sigma \wedge \lambda)=\phi(a)\left[\sigma\left(\tilde{u}_{s}\right)^{2}+\lambda\left(\tilde{u}_{s}\right)^{2}\right] d s \wedge d t .
\end{gathered}
$$

Thus, we have $E_{\omega}(\tilde{u}) \geq 0$ and $E_{\lambda}(\tilde{u}) \geq 0$.

2C. Main results. The next two theorems tell us the behaviors of $J$-holomorphic curves near infinity.

Theorem 2.8. Suppose that $J$ is an asymptotically cylindrical almost complex structure on $\mathbb{R}^{ \pm} \times V$ at $\pm \infty$, and suppose that $J$ is of the Morse-Bott type. Let $\tilde{u}=(a, u): \mathbb{R}^{ \pm} \times \mathbb{R} / \mathbb{Z} \rightarrow \mathbb{R}^{ \pm} \times V$ be a finite energy J-holomorphic curve. Suppose that the image of $\tilde{u}$ is unbounded in $\mathbb{R}^{ \pm} \times V$. Then there exists a periodic orbit $\gamma$ of $\boldsymbol{R}_{\infty}$ of period $|T|$ with $T \neq 0$ such that, in $C^{\infty}\left(S^{1}\right)$,

$$
\lim _{s \rightarrow \pm \infty} u(s, t)=\gamma(T t) \quad \text { and } \quad \lim _{s \rightarrow \pm \infty} \frac{a(s, t)}{s}=T .
$$

The above theorem tells us that when $|s|$ is large enough $u(s, t)$ lies inside a small neighborhood of $\gamma$. We will construct a coordinate chart for such a neighborhood $U \subset S^{1} \times \mathbb{R}^{2 n} \rightarrow V$, and then we can view the map $\tilde{u}$ as

$$
\tilde{u}(s, t)=(a(s, t), \vartheta(s, t), z(s, t)) \in \mathbb{R}^{ \pm} \times \mathbb{R} \times \mathbb{R}^{2 n},
$$

where $\vartheta$ is the coordinate of the universal cover of $S^{1}=\mathbb{R} / \mathbb{Z}$.

Theorem 2.9. Under the same assumption as in Theorem 2.8, there exist constants $M_{\beta}, d_{\beta}, a_{0}, \vartheta_{0}, s_{0}>0$ such that

$$
\begin{aligned}
\left|D^{\beta}\left\{a(s, t)-T s-a_{0}\right\}\right| & \leq M_{\beta} e^{\mp d_{\beta} s}, \\
\left|D^{\beta}\left\{\vartheta(s, t)-T t-\vartheta_{0}\right\}\right| & \leq M_{\beta} e^{\mp d_{\beta} s}, \\
\left|D^{\beta} z(s, t)\right| & \leq M_{\beta} e^{\mp d_{\beta} s},
\end{aligned}
$$

for all $s>s_{0}$ and $\beta=\left(\beta_{1}, \beta_{2}\right) \in \mathbb{Z}_{\geq 0} \times \mathbb{Z}_{\geq 0}$.

\section{Proof of main results}

The proofs for $\mathbb{R}^{+} \times V$ and $\mathbb{R}^{-} \times V$ are almost the same, so we will focus on the $\mathbb{R}^{+} \times V$ case. The proof is done in three steps. The first step is to show that the gradient of a finite Hofer energy $J$-holomorphic curve $\tilde{u}=(a, u)$ is bounded. The second step is to show "subsequence convergence": briefly, given a sequence of numbers $R_{k}$ converging to infinity, we want to show that there exists a subsequence $R_{k_{n}}$ such 
that $u\left(R_{k_{n}}, t\right)$ converges to a periodic solution of the vector field $\boldsymbol{R}_{\infty}$. The third step is to get an exponential decay estimate and then prove Theorems 2.8 and 2.9.

3A. Gradient bounds. We cite the following two lemmata for later use.

Lemma 3.1 [Hofer 1993]. Let $(X, d)$ be a metric space. The following statements are equivalent:

(a) $(X, d)$ is complete.

(b) For every continuous map $\phi: X \rightarrow[0,+\infty)$ and a given $x \in X, \varepsilon>0$ there exist $x^{\prime} \in X, \varepsilon^{\prime}>0$ such that

- $\varepsilon^{\prime} \leq \varepsilon, \phi\left(x^{\prime}\right) \varepsilon^{\prime} \geq \phi(x) \varepsilon$

- $d\left(x, x^{\prime}\right) \leq 2 \varepsilon$,

- $2 \phi\left(x^{\prime}\right) \geq \phi(y)$ for all $y \in X$ with $d\left(y, x^{\prime}\right) \leq \varepsilon^{\prime}$.

Let $J$ be an asymptotically cylindrical almost complex structure on $W=\mathbb{R}^{+} \times V$ at $\infty$, and let $\tilde{u}=(a, u)$ be a $J$-holomorphic map from $B(0, R)$ to $W$, where $B\left(z_{0}, R\right):=z=\left\{s+\sqrt{-1} t \in \mathbb{C}:\left|z-z_{0}\right|<R\right\}$. Define

$$
\|\nabla \tilde{u}\|:=\sup _{(s, t) \in B(0, R)}|\nabla \tilde{u}(s, t)|
$$

and

$$
\|\tilde{u}\|_{C^{k}(B(0, R), W)}:=\sup _{x \in B(0, R)} \sum_{|l|=0}^{k}\left|\nabla^{l} \tilde{u}(x)\right|,
$$

where the norm $|\cdot|$ is taken with respect to the standard metric $d s^{2}+d t^{2}$ on $B\left(z_{0}, R\right)$ and to a translationally invariant metric $g_{W}$ on $W$ (for example, $g_{W}=g_{V}+d r^{2}$ ), and $\nabla$ is the Levi-Civita connection with respect to $g_{W}$ on $W$. The following lemma says that the gradient bound implies a $C^{\infty}$ bound.

Lemma 3.2 (Gromov-Schwarz). Fix $0<\varepsilon<1$ and $k \in \mathbb{N}$. If $\|\nabla \tilde{u}\|<C^{\prime}<+\infty$, then there exists a $C\left(k, C^{\prime}\right)>0$ such that

$$
\|\tilde{u}\|_{C^{k}(B(0, R-\varepsilon), W)} \leq C\left(k, C^{\prime}\right),
$$

where $C\left(k, C^{\prime}\right)$ does not depend on $\tilde{u}$.

Proof. This is a standard result. Using the gradient bound of $\tilde{u}$, we can find uniform coordinate charts both in domain and in target, then we can apply Proposition 2.36 in [Audin and Lafontaine 1994].

The following proposition, whose proof reveals the relation between the $\omega$-energy and trajectory of $\boldsymbol{R}_{\infty}$, is one of the key steps in [Hofer 1993].

Proposition 3.3 [Hofer 1993]. Suppose $J$ is a cylindrical almost complex structure on $\mathbb{R} \times V$ and let $\tilde{u}=(a, u): \mathbb{C} \rightarrow \mathbb{R} \times V$ be a finite Hofer energy J-holomorphic 
plane (i.e., $\left.E(\tilde{u})=E_{\lambda}(\tilde{u})+E_{\omega}(\tilde{u})<+\infty\right)$. If $E_{\omega}(\tilde{u})=0$ and $\|\nabla \tilde{u}\| \leq C$ for some $C>0$, then $\tilde{u}$ is constant.

Proof. Suppose $\tilde{u}$ is not constant. By (3), $\pi_{\xi} \tilde{u}_{s}=0=\pi_{\xi} \tilde{u}_{t}$. Hence $\pi_{\xi} \circ T \tilde{u}$ is the zero section of $\tilde{u}^{*} \xi \rightarrow \mathbb{C}$. Therefore we have $u(s, t)=x \circ f(s, t)$, where $x: \mathbb{R} \rightarrow V$ satisfies $\dot{x}=\boldsymbol{R}(x)$ and $f: \mathbb{C} \rightarrow \mathbb{R}$ is a smooth function. Consequently, $f_{s}=-a_{t}$ and $f_{t}=a_{s}$. Hence $\Phi:=f+i a$ is a holomorphic function on $\mathbb{C}$. Since $\|\nabla \tilde{u}\|$ is bounded, $\|\nabla \Phi\|$ is bounded; thus $\Phi$ is a linear function. By (4),

$$
E_{\lambda}(\tilde{u})=\sup _{\phi \in \mathcal{C}} \int_{\mathbb{C}} \phi(a)\left(a_{s}^{2}+a_{t}^{2}\right) d s \wedge d t=+\infty,
$$

via a linear change of variables.

The proposition below generalizes Proposition 27 in [Hofer 1993] to the asymptotically cylindrical case.

Proposition 3.4. If $J$ is an asymptotically cylindrical almost complex structure on $W=\mathbb{R}^{+} \times V$ at $\infty$, and $\tilde{u}$ is a J-holomorphic map from $\mathbb{C}$ to $W$ satisfying $E(\tilde{u})<+\infty$, then $\|\nabla \tilde{u}\|<+\infty$.

Proof. Suppose to the contrary that there exists a sequence of points $z_{k} \in \mathbb{C}$ satisfying $\left|z_{k}\right| \rightarrow \infty, R_{k}:=\left\|\nabla \tilde{u}\left(z_{k}\right)\right\| \rightarrow \infty$, as $k \rightarrow \infty$. By Lemma 3.1, we can modify $z_{k}$ such that there exists a sequence of $\varepsilon_{k}>0$ satisfying $\varepsilon_{k} \rightarrow 0, \varepsilon_{k} R_{k} \rightarrow+\infty$, and $|\nabla \tilde{u}(z)| \leq 2 R_{k}$ for $z \in B\left(z_{k}, \varepsilon_{k}\right)$. Now there are two cases.

Case 1: $\left\{a\left(z_{k}\right)\right\}_{k \in \mathbb{Z}}$ is unbounded.

Then there exists a subsequence of $z_{k}$, still denoted by $z_{k}$, such that $a\left(z_{k}\right) \rightarrow+\infty$ or $a\left(z_{k}\right) \rightarrow-\infty$. Without loss of generality, let's assume $a\left(z_{k}\right) \rightarrow+\infty$. Pick a further subsequence of $z_{k}$ such that $a\left(z_{k}\right) \geq 2^{k+2}$. Let $\varepsilon_{k}^{\prime}:=\min \left\{\varepsilon_{k}, 2^{k} / R_{k}\right\}$. Then we have $\varepsilon_{k}^{\prime} \rightarrow 0, \varepsilon_{k}^{\prime} R_{k} \rightarrow+\infty$, and $\left|a(z)-a\left(z_{k}\right)\right| \leq 2 \varepsilon_{k}^{\prime} R_{k} \leq 2\left(2^{k} / R_{k}\right) R_{k}=2^{k+1}$, for $\left|z-z_{k}\right| \leq \varepsilon_{k}^{\prime}$. Thus, $a(z) \geq a\left(z_{k}\right)-2^{k+1} \geq 2^{k+2}-2^{k+1}=2^{k+1}$, for $\left|z-z_{k}\right| \leq \varepsilon_{k}^{\prime}$.

Since $\tilde{u}$ is $J$-holomorphic, we have

$$
J(\tilde{u}) \circ T \tilde{u}=T \tilde{u} \circ i .
$$

Thus

$$
J_{\infty}(\tilde{u}) \circ T \tilde{u}=T \tilde{u} \circ i+\left(J_{\infty}-J\right)(\tilde{u}) \circ T \tilde{u} .
$$

By (AC1), we have, as $k \rightarrow+\infty,{ }^{2}$

$$
\sup _{z \in B\left(z_{k}, \varepsilon_{k}^{\prime}\right)}\left\|\left(J_{\infty}-J\right)(\tilde{u}(z))\right\| \rightarrow 0 .
$$

${ }^{2}$ Actually, to prove Proposition 3.4, Proposition 3.5 and Theorem 3.7 we only need $f_{s}^{*} J \rightarrow J_{\infty}$ in $C_{\text {loc }}^{1}$ as $s \rightarrow \infty$. We need the stronger condition (AC1) to prove exponential decay in Section 3C and thus the main theorems. 
Define maps $\tilde{u}_{k}(z)=\left(a\left(z_{k}+z / R_{k}\right)-a\left(z_{k}\right), u\left(z_{k}+z / R_{k}\right)\right)$ from $\mathbb{C}$ to $\mathbb{R} \times V$. For any $R^{\prime}>0$, when $k$ is large, $\left\|\nabla \tilde{u}_{k}(z)\right\| \leq 2$ for $z \in B\left(0, R^{\prime}\right)$. By Lemma 3.2, for any $n \in \mathbb{Z}_{\geq 0}$, there exists a $C\left(n, R^{\prime}\right)$ satisfying

$$
\left\|\tilde{u}_{k}\right\|_{C^{n}\left(B\left(0, R^{\prime}-1\right), W\right)} \leq C\left(n, R^{\prime}\right)
$$

We also have

$$
\begin{gathered}
\left|\nabla \tilde{u}_{k}(0)\right|=1, \\
\left|\nabla \tilde{u}_{k}(z)\right| \leq 2 \text { for all }|z| \leq \varepsilon_{k}^{\prime} R_{k} .
\end{gathered}
$$

We apply the Ascoli-Arzela theorem to get a subsequence, still called $\tilde{u}_{k}$, satisfying $\tilde{u}_{k} \rightarrow \tilde{u}_{\infty}$ in $C_{\text {loc }}^{\infty}$ as $k \rightarrow \infty$. Here $\tilde{u}_{\infty}: \mathbb{C} \rightarrow \mathbb{R} \times V$ is a $J_{\infty}$-holomorphic map satisfying

$$
\left|\nabla \tilde{u}_{\infty}(0)\right|=1 \quad \text { and } \quad\left\|\nabla \tilde{u}_{\infty}\right\| \leq 2 .
$$

Indeed, $\tilde{u}_{k}$ satisfies

$$
J_{\infty}\left(\tilde{u}_{k}\right) T \tilde{u}_{k}=T \tilde{u}_{k} i+o_{k},
$$

where $\left\|o_{k}\right\|_{C^{0}\left(B\left(0, \varepsilon_{k}^{\prime} R_{k}\right)\right)} \rightarrow 0$ as $k \rightarrow \infty$. Therefore, $\tilde{u}_{\infty}$ is $J_{\infty}$-holomorphic.

Now let's look at its energy:

$$
\int_{B\left(0, R^{\prime}\right)} \tilde{u}_{k}^{*} \omega_{\infty}=\int_{B\left(z_{k}, R^{\prime} / R_{k}\right)} \tilde{u}^{*} \omega+\int_{B\left(z_{k}, R^{\prime} / R_{k}\right)} \tilde{u}^{*}\left(\omega-\omega_{\infty}\right) .
$$

From $E(\tilde{u})<+\infty$ we see that $\int_{B\left(z_{k}, R^{\prime} / R_{k}\right)} \tilde{u}^{*} \omega \rightarrow 0$ as $k \rightarrow+\infty$. We also have

$$
\begin{aligned}
\left|\int_{B\left(z_{k}, R^{\prime} / R_{k}\right)} \tilde{u}^{*}\left(\omega_{\infty}-\omega\right)\right| & \leq \int_{B\left(z_{k}, R^{\prime} / R_{k}\right)}\left(2 R_{k}\right)^{2}\left|\left(\omega_{\infty}-\omega\right)\left(\frac{\tilde{u}_{s}}{2 R_{k}}, \frac{\tilde{u}_{t}}{2 R_{k}}\right)\right| d s \wedge d t \\
& \leq \pi\left(\frac{R^{\prime}}{R_{k}}\right)\left(2 R_{k}\right)^{2} c_{k} \rightarrow 0,
\end{aligned}
$$

where

$$
c_{k}:=\sup _{z \in B\left(z_{k}, \varepsilon_{k}^{\prime}\right)}\left|\left(\omega_{\infty}-\omega\right)\left(\frac{\tilde{u}_{s}}{2 R_{k}}, \frac{\tilde{u}_{t}}{2 R_{k}}\right)\right|,
$$

and by (AC4) $c_{k} \rightarrow 0$ as $k \rightarrow \infty$. Therefore,

$$
E_{\omega_{\infty}}\left(\tilde{u}_{\infty}\right)=\int_{\mathbb{C}} \tilde{u}_{\infty}^{*} \omega_{\infty}=0
$$


Moreover, we have $E_{\lambda_{\infty}}\left(\tilde{u}_{\infty}\right)<+\infty$. Given $\phi \in \mathcal{C}$, define $\phi_{k}(r):=\phi\left(r-a\left(z_{k}\right)\right) \in \mathcal{C}$. Then we have

$$
\begin{aligned}
& \left|\int_{B\left(0, R^{\prime}\right)} \tilde{u}_{k}^{*}\left(\phi(r) d r \wedge \lambda_{\infty}\right)\right| \\
& \quad \leq\left|\int_{B\left(z_{k}, R^{\prime} / R_{k}\right)} \phi_{k}(a) \tilde{u}^{*}(\sigma \wedge \lambda)\right|+\left|\int_{B\left(z_{k}, R^{\prime} / R_{k}\right)} \phi_{k}(a) \tilde{u}^{*}\left(d r \wedge \lambda_{\infty}-\sigma \wedge \lambda\right)\right| .
\end{aligned}
$$

We also have

$$
\left|\int_{B\left(z_{k}, R^{\prime} / R_{k}\right)} \phi_{k}(a) \tilde{u}^{*}(\sigma \wedge \lambda)\right| \leq\left|\int_{\mathbb{C}} \phi_{k}(a) \tilde{u}^{*}(\sigma \wedge \lambda)\right| \leq E_{\lambda}(\tilde{u})
$$

and

$$
\begin{aligned}
& \left|\int_{B\left(z_{k}, R^{\prime} / R_{k}\right)} \phi_{k}(a) \tilde{u}^{*}\left(d r \wedge \lambda_{\infty}-\sigma \wedge \lambda\right)\right| \\
& \quad \leq \int_{B\left(z_{k}, R^{\prime} / R_{k}\right)} \phi_{k}(a)\left(2 R_{k}\right)^{2}\left|\left(d r \wedge \lambda_{\infty}-\sigma \wedge \lambda\right)\left(\frac{\tilde{u}_{s}}{2 R_{k}}, \frac{\tilde{u}_{t}}{2 R_{k}}\right)\right| d s \wedge d t \\
& \left.\quad \leq \sup _{x \in \mathbb{R}} \phi(x)\right)\left(2 R_{k}\right)^{2} r_{k} \pi\left(\frac{R^{\prime}}{R_{k}}\right)^{2} \rightarrow 0,
\end{aligned}
$$

where

$$
r_{k}:=\sup _{z \in B\left(z_{k}, R^{\prime} / R_{k}\right)}\left|\left(d r \wedge \lambda_{\infty}-\sigma \wedge \lambda\right)\left(\frac{\tilde{u}_{s}}{2 R_{k}}, \frac{\tilde{u}_{t}}{2 R_{k}}\right)\right| \rightarrow 0
$$

as $k \rightarrow \infty$. Combining (13), (14) and (15), we get the following result: given $R^{\prime}>0$ and $\phi \in \mathcal{C}$, there exists a constant $K$ such that, for all $k>K$,

$$
\left|\int_{B\left(0, R^{\prime}\right)} \tilde{u}_{k}^{*}\left(\phi(r) d r \wedge \lambda_{\infty}\right)\right| \leq E_{\lambda}(\tilde{u})+1 .
$$

Therefore, $E_{\lambda_{\infty}}\left(\tilde{u}_{\infty}\right) \leq E_{\lambda}(\tilde{u})+1$. Altogether, we get a $J_{\infty}$-holomorphic map $\tilde{u}_{\infty}: \mathbb{C} \rightarrow W$ satisfying

$$
\left\|\nabla \tilde{u}_{\infty}\right\| \leq 2, \quad\left|\nabla \tilde{u}_{\infty}(0)\right|=1, \quad E_{\omega_{\infty}}\left(\tilde{u}_{\infty}\right)=0, \quad E\left(\tilde{u}_{\infty}\right)<+\infty .
$$

By Proposition 3.3, we get a contradiction, which finishes the proof for Case 1 .

Case 2: $\left\{a\left(z_{k}\right)\right\}_{k \in \mathbb{Z}}$ is bounded.

Now let us define $\tilde{u}_{k}$, differently from Case 1 , by

$$
\tilde{u}_{k}(z):=\tilde{u} \circ l_{k}=\left(a\left(z_{k}+z / R_{k}\right), u\left(z_{k}+z / R_{k}\right)\right) .
$$


Then $\tilde{u}_{k}$ satisfies

$$
\begin{gathered}
\left|\nabla \tilde{u}_{k}(z)\right| \leq 2 \text { for } z \in B\left(0, \varepsilon_{k} R_{k}\right), \\
\left\{\tilde{u}_{k}(0)\right\}_{k \in \mathbb{Z}^{+}} \text {is bounded, } \\
|\nabla \tilde{u}(0)|=1 .
\end{gathered}
$$

Similar to Case 1, by applying the Ascoli-Arzela theorem we get a subsequence, still called $\tilde{u}_{k}$, converging to $\tilde{u}_{\infty}=\left(a_{\infty}, u_{\infty}\right): \mathbb{C} \rightarrow W$ in the $C_{\text {loc }}^{\infty}$ sense. Here $\tilde{u}_{\infty}$ is $J$-holomorphic, satisfying

$$
\begin{gathered}
\left|\nabla \tilde{u}_{\infty}(0)\right|=1, \\
\left\|\nabla \tilde{u}_{\infty}\right\| \leq 2, \\
\int_{B\left(0, \varepsilon_{k} R_{k}\right)} \tilde{u}_{k}^{*} \omega=\int_{B\left(z_{k}, \varepsilon_{k}\right)} \tilde{u}^{*} \omega \rightarrow 0 \quad \text { as } k \rightarrow+\infty .
\end{gathered}
$$

Thus, $E_{\omega}\left(\tilde{u}_{\infty}\right)=\int_{\mathbb{C}} \tilde{u}_{\infty}^{*} \omega=0$. Moreover, given $R^{\prime}>0$ and $\phi \in \mathcal{C}$, we have

$$
\int_{B\left(0, R^{\prime}\right)} \tilde{u}_{k}^{*}[\phi(r) \sigma \wedge \lambda]=\int_{B\left(z_{k}, R^{\prime} / R_{k}\right)} \tilde{u}^{*}[\phi(r) \sigma \wedge \lambda] \rightarrow 0
$$

as $k \rightarrow+\infty$. This means $\int_{B\left(0, R^{\prime}\right)} \tilde{u}_{\infty}^{*}[\phi(r) \sigma \wedge \lambda]=0$, and so $E_{\lambda}\left(\tilde{u}_{\infty}\right)=0$. Hence, $\tilde{u}_{\infty}$ is constant, contradicting (16).

Proposition 3.5. Suppose $J$ is a cylindrical almost complex structure on $\mathbb{R} \times V$. Let $\tilde{v}: \mathbb{R}^{+} \times S^{1} \rightarrow W$ be a J-holomorphic map with respect to the standard complex structure on $\mathbb{R}^{+} \times S^{1}$, and assume $E(\tilde{v})<+\infty$. Then we have

$$
\|\nabla \tilde{v}\|<+\infty, \quad \text { where }\|\nabla \tilde{v}\|:=\sup _{(s, t) \in \mathbb{R}^{+} \times S^{1}}|\nabla \tilde{v}(s, t)|,
$$

and the norm $|\cdot|$ is taken with respect to the standard metric $d s^{2}+d t^{2}$ on $\mathbb{R}^{+} \times S^{1}$ and to a translationally invariant metric $g_{W}$ on $W$, and $\nabla$ is the Levi-Civita connection with respect to $g_{W}$.

Proof. The proof is almost the same as the proof of Proposition 3.4.

Remark 3.6. Actually, we can see that we can get a gradient bound with respect to a metric $g_{D}$ on the domain and a translationally invariant metric $g_{W}$ on $W$, as long as the injectivity radius of $g_{D}$ is bounded away from 0 .

\section{B. Subsequence convergence.}

Theorem 3.7. Let $J$ be an asymptotically cylindrical almost complex structure on $\mathbb{R}^{ \pm} \times V$, and let $\tilde{v}=(a, v): \mathbb{R}^{ \pm} \times S^{1} \rightarrow \mathbb{R}^{ \pm} \times V$ be a J-holomorphic curve with $E(\tilde{v})<+\infty$. Suppose that $\tilde{v}\left(\mathbb{R}^{ \pm} \times S^{1}\right)$ is unbounded. Then for any sequence 
$k_{n} \rightarrow+\infty$, there exists a subsequence $k_{n_{i}}$ such that $v\left(k_{n_{i}}, \cdot\right)$ converges in $C^{\infty}\left(S^{1}\right)$ to a map $S^{1} \rightarrow V$ given by $t \mapsto x(t T)$, where $x: \mathbb{R} \rightarrow V$ is a $|T|$-periodic solution of $\dot{x}=\boldsymbol{R}_{\infty}(x)$.

Proof. We prove this theorem for the case $\mathbb{R}^{+} \times V$. The proof for the $\mathbb{R}^{-} \times V$ case can be carried out similarly, and hence is omitted. By Proposition 3.5 we have $\|\nabla \tilde{v}\| \leq C$ for some $C>0$. Since $\tilde{v}\left(\mathbb{R}^{+} \times S^{1}\right)$ is not bounded, there exists a sequence of points $\left(s_{k}, t_{k}\right) \in \mathbb{R}^{+} \times S^{1}$ such that $\left|a\left(s_{k}, t_{k}\right)\right| \rightarrow+\infty$. Now there are two cases.

Case 1: $a\left(s_{k}, t_{k}\right) \rightarrow+\infty$.

Suppose that there exists a sequence of points $\left(s_{k}^{\prime}, t_{k}^{\prime}\right) \in \mathbb{R}^{+} \times S^{1}$ such that $a\left(s_{k}^{\prime}, t_{k}^{\prime}\right)<Q$ for some constant $Q$. Pick a subsequence of $\left(s_{k}, t_{k}\right)$, still called $\left(s_{k}, t_{k}\right)$, and a subsequence of $\left(s_{k}^{\prime}, t_{k}^{\prime}\right)$, still called $\left(s_{k}^{\prime}, t_{k}^{\prime}\right)$, so that they satisfy $s_{k}^{\prime}<s_{k}<s_{k+1}^{\prime}$ for all $k$. This is possible because $s_{k} \rightarrow+\infty$. Since $\|\nabla \tilde{v}\| \leq C$, we have $a\left(s_{k}^{\prime}, t\right)<Q+C$ for $t \in S^{1}$. Consider the compact manifold $N:=[Q, Q+2 C] \times M \subset W=\mathbb{R}^{+} \times V$. Pick a $\phi \in \mathcal{C}$ such that $\left.\phi\right|_{[Q, Q+2 C]}>0$. By Gromov's monotonicity theorem (see for example Theorem 1.3 in [Hummel 1997]), there exists an $\iota>0$ such that

$$
\int_{\left.\left., s_{k}\right] \times S^{1}\right)} \omega+\phi(r) \sigma \wedge \lambda \geq \iota>0
$$

for all $k$. This contradicts the fact that $E(\tilde{v})<+\infty$. Thus $a(s, t) \rightarrow+\infty$ uniformly in $t$ as $s \rightarrow+\infty$.

Define

$$
\tilde{v}_{n}(s, t)=\left(a\left(s+k_{n}, t\right)-a\left(k_{n}, 0\right), v\left(s+k_{n}, t\right)\right) .
$$

Then the sequence $\tilde{v}_{n}(0,0)=\left(0, v\left(k_{n}, 0\right)\right)$ is bounded. Since $\tilde{v}$ is $J$-holomorphic, by Lemma 3.2 and the Ascoli-Arzela theorem, there exists a subsequence, still called $\tilde{v}_{n}$, converging to $\tilde{v}_{\infty}=\left(b, v_{\infty}\right): \mathbb{R} \times S^{1} \rightarrow W$ in $C_{\text {loc }}^{\infty}$. We know $\tilde{v}_{\infty}$ is $J_{\infty}$-holomorphic. Define the translation map $\tau_{n}: \mathbb{R} \times S^{1} \rightarrow \mathbb{R} \times S^{1}$ by $\tau_{n}(s, t)=\left(s+k_{n}, t\right)$. Now observe that

$$
\int_{[-R, R] \times S^{1}} \tilde{v}_{n}^{*} \omega_{\infty}=\int_{\left[-R+k_{n}, R+k_{n}\right] \times S^{1}} \tilde{v}^{*} \omega+\int_{\left[-R+k_{n}, R+k_{n}\right] \times S^{1}} \tilde{v}^{*}\left(\omega_{\infty}-\omega\right) .
$$

For the first term on the right-hand side we have

$$
\int_{\left[-R+k_{n}, R+k_{n}\right] \times S^{1}} \tilde{v}^{*} \omega \rightarrow 0
$$

as $n \rightarrow \infty$, since $E_{\omega}(\tilde{v})$ is finite. By (AC4), the second term satisfies, as $n \rightarrow+\infty$,

$$
\int_{\left[-R+k_{n}, R+k_{n}\right] \times S^{1}} \tilde{v}^{*}\left(\omega_{\infty}-\omega\right) \leq \int_{\left[-R+k_{n}, R+k_{n}\right] \times S^{1}}\left|\left(\omega_{\infty}-\omega\right)\left(\tilde{v}_{s}, \tilde{v}_{t}\right)\right| d s \wedge d t \rightarrow 0 .
$$


Combining (19), (20) and (21), we can see that $\int_{[-R, R] \times S^{1}} \tilde{v}_{\infty}^{*} \omega_{\infty}=0$ and hence $E_{\omega_{\infty}}\left(\tilde{v}_{\infty}\right)=0$, so there exists a smooth map $f: \mathbb{R}^{2} \rightarrow \mathbb{R}$ such that $\tilde{v}_{\infty}=(b, x \circ f)$, where $x: \mathbb{R} \rightarrow V$ is the solution of $\dot{x}=\boldsymbol{R}_{\infty}(x)$. Let $\Phi$ be the holomorphic function defined by $\Phi=b+i f$. Since $\|\nabla \Phi\| \leq C$, we know that $\Phi$ is linear. Thus, $\Phi(s, t)=\alpha(s+i t)+\beta$, where $\alpha=T+i l, \beta=m+i n \in \mathbb{C}$ are constants. But $b(s, t)-b(s, t+1)=0$ implies $l=0$, and $b(0,0)=0$ implies $m=0$. Thus,

$$
\begin{gathered}
f=T t+n, \\
b=T s .
\end{gathered}
$$

Therefore, $a_{s}\left(k_{n}, t\right) \rightarrow T$ uniformly in $t$ as $n \rightarrow+\infty$ (recall the notation $\tilde{v}=(a, v)$, $\left.\tilde{v}_{\infty}=\left(b, v_{\infty}\right)\right)$. Moreover, we have

$$
\int_{\{0\} \times S^{1}} \tilde{v}_{\infty}^{*} \lambda_{\infty}=\int_{\{0\} \times S^{1}} \lambda_{\infty}\left[\left(\tilde{v}_{\infty}\right)_{t}\right] d t=\int_{\{0\} \times S^{1}} b_{s} d t=T .
$$

Claim: $T \neq 0$.

It follows from the claim and (22) that $\tilde{v}_{\infty}$ is not constant. Indeed, by (22), $f(s, t+1)=T(t+1)+n$, so $x(T(t+1)+n)=x(T t+n)$. Hence, $x$ is $T$-periodic.

Proof. Suppose $T=0$. Since $a(s, t) \rightarrow+\infty$ uniformly in $t$ as $s \rightarrow+\infty$, we can choose a subsequence $k_{n_{m}}$ of $k_{n}$ and a sequence $t_{m} \in S^{1}$ so that we have $a\left(k_{n_{m+1}}, t_{m+1}\right)-a\left(k_{n_{m}}, t_{m}\right) \geq 4 C$. Denote $a\left(k_{n_{m}}, t_{m}\right)$ by $a_{m}$. Then from $\|\nabla \tilde{u}\| \leq C$ we get

$$
\begin{gathered}
a\left(k_{n_{m}}, t\right) \in\left[a_{m}-C, a_{m}+C\right], \\
a\left(k_{n_{m+1}}, t\right) \geq a_{m}+3 C .
\end{gathered}
$$

Let $\psi_{m}: \mathbb{R} \rightarrow[0,1]$ be a smooth map, satisfying $\psi_{m}(r)=\frac{1}{7 C}\left(r-a_{m}+\frac{3}{2} C\right)$ for $r \in\left[a_{m}-C, a_{m}+5 C\right]$ and $\phi_{m}=\psi_{m}^{\prime} \in \mathcal{C}$. If we further require $C>1$, then $\phi_{m}(r) \leq \frac{1}{7 C}<1$. Observe that

$$
\int_{\left[k_{n_{m}}, k_{n_{m+1}}\right] \times S^{1}} \tilde{v}^{*} d\left(\psi_{m}(r) \lambda\right)=\int_{\left\{k_{n_{m+1}}\right\} \times S^{1}} \tilde{v}^{*}\left(\psi_{m}(r) \lambda\right)-\int_{\left\{k_{n_{m}}\right\} \times S^{1}} \tilde{v}^{*}\left(\psi_{m}(r) \lambda\right) .
$$

We also have, as $m \rightarrow+\infty$,

$\left|\int_{\left\{k_{n_{m+1}}\right\} \times S^{1}} \tilde{v}^{*}\left(\psi_{m}(r) \lambda\right)\right|=\left|\int_{\left\{k_{n_{m+1}}\right\} \times S^{1}} \psi_{m}(\tilde{v}) \lambda\left(\tilde{v}_{t}\right) d t\right| \leq \int_{\left\{k_{n_{m+1}}\right\} \times S^{1}}\left|\lambda\left(\tilde{v}_{t}\right)\right| d t \rightarrow T=0$. 
Similarly, $\int_{\left\{k_{n_{m}}\right\} \times S^{1}} \tilde{v}^{*}\left(\psi_{m}(r) \lambda\right) \rightarrow 0$. Thus, by Stokes' theorem,

$$
\int_{\left.k_{n_{m+1}}\right] \times S^{1}} \tilde{v}^{*} d\left(\psi_{m}(r) \lambda\right) \rightarrow 0
$$

Observe that

$$
\begin{aligned}
\int & \tilde{v}^{*}\left(\phi_{m}(r) \sigma \wedge \lambda\right) \\
& =\int_{\left[k_{n_{m}}, k_{n_{m+1}}\right] \times S^{1}} \tilde{v}^{*}\left(\phi_{m}(r) d r \wedge \lambda\right)+\int_{\left[k_{n_{m}}, k_{n_{m+1}}\right] \times S^{1}} \tilde{v}^{*}\left[\phi_{m}(r)(\sigma-d r) \wedge \lambda\right] .
\end{aligned}
$$

For the first term on the right-hand side, we have, for some $c>0, c_{m}>0$,

$$
\begin{aligned}
& \left|\int_{\left[k_{n_{m}}, k_{n_{m+1}}\right] \times S^{1}} \tilde{v}^{*}\left(\phi_{m}(r) d r \wedge \lambda\right)\right| \\
& \leq\left|\int_{\left[k_{n_{m}}, k_{n_{m+1}}\right] \times S^{1}} \tilde{v}^{*} d\left(\psi_{m}(r) \lambda\right)\right|+\int_{\left[k_{n_{m}}, k_{n_{m+1}}\right] \times S^{1}}\left|\tilde{v}^{*}\left(\psi_{m}(r) d \lambda\right)\right| \\
& \leq\left|\int_{\left[k_{n_{m}}, k_{n_{m+1}}\right] \times S^{1}} \tilde{v}^{*} d\left(\psi_{m}(r) \lambda\right)\right|+\int_{\left[k_{n_{m}}, k_{n_{m+1}}\right] \times S^{1}} \tilde{v}^{*}\left(c \omega+c_{m} \sigma \wedge \lambda\right) .
\end{aligned}
$$

The second inequality is due to the fact that $c \omega+c_{m} \sigma \wedge \lambda$ is positive on all $J$-complex planes; also since $d \lambda \rightarrow d \lambda_{\infty}$ and $i(\partial / \partial r) d \lambda_{\infty}=0=i\left(\boldsymbol{R}_{\infty}\right) d \lambda_{\infty}$, we can require that $c$ is independent of $m$ and $c_{m}$ goes to 0 as $m \rightarrow+\infty$. Similarly, we have

$$
\left|\int_{\left[k_{n_{m}}, k_{n_{m+1}}\right] \times S^{1}} \tilde{v}^{*}\left[\phi_{m}(r)(\sigma-d r) \wedge \lambda\right]\right| \leq \int_{\left[k_{n_{m}}, k_{n_{m+1}}\right] \times S^{1}} \tilde{v}^{*}\left[c \omega+c_{m} \sigma \wedge \lambda\right] .
$$

When $k$ is large, from (28), (29) and (30) we get

$$
\int_{\left[k_{n_{m}}, k_{n_{m+1}}\right] \times S^{1}} \tilde{v}^{*}\left(\phi_{m}(r) \sigma \wedge \lambda\right) \leq D\left\{\left|\int_{\left[k_{n_{m}}, k_{n_{m+1}}\right] \times S^{1}} \tilde{v}^{*} d\left(\psi_{m}(r) \lambda\right)\right|+\int_{\left[k_{n_{m}}, k_{n_{m+1}}\right] \times S^{1}} \tilde{v}^{*} \omega\right\},
$$

for some constant $D>0$ which does not depend on $m$ and $\tilde{v}$. The term

$$
\int_{\left[k_{n_{m}}, k_{n_{m+1}}\right] \times S^{1}} \tilde{v}^{*}\left(c_{m} \sigma \wedge \lambda\right)
$$


does not show up on the right-hand side of (31) because it is absorbed by the left-hand side, since $\left.\phi_{m}\right|_{\left[k_{n_{m}}, k_{n_{m+1}}\right] \times S^{1}}=1 / 7$. Since $E_{\omega}(\tilde{v})$ is finite,

$$
\int_{\left[k_{n_{m}}, k_{n_{m+1}}\right] \times S^{1}} \tilde{v}^{*} \omega \rightarrow 0 .
$$

Together with (27), we get

$$
\int_{\left., k_{n_{m+1}}\right] \times S^{1}} \tilde{v}^{*}\left(\phi_{m}(r) d r \wedge \lambda\right) \rightarrow 0 .
$$

Summing up, we have, as $m \rightarrow+\infty$,

$$
\int_{\left[k_{n_{m}}, k_{n_{m+1}}\right] \times S^{1}} \tilde{v}^{*}\left(\omega+\phi_{m}(r) d r \wedge \lambda\right) \rightarrow 0 .
$$

Now consider $N_{m}=\left[a_{m}+C, a_{m}+3 C\right] \times V \subseteq W$ with an almost complex structure $J_{m}:=\left.J\right|_{N_{m}}$ and a nondegenerate 2-form $\Omega_{m}:=\omega+\left.\phi_{m}(r) \sigma \wedge \lambda\right|_{N_{m}}$. Because of the asymptotic condition, we can find uniform constants $C_{0}, r_{0}>0$ such that by Gromov's monotonicity theorem, for any $J_{m}$-holomorphic curve $h_{m}:(S, j) \rightarrow\left(N_{m}, J_{m}\right)$, where $(S, j)$ is a Riemann surface with boundary, if the boundary $h_{m}(\partial S)$ is contained in the complement of the ball $B\left(h_{m}\left(s_{0}\right), r\right)$, where $s_{0} \in \operatorname{Int} S_{m}$ and $r<r_{0}$, then we have

$$
\int_{\left.h_{m}(S) \cap B\left(h_{m}\left(s_{0}\right), r\right)\right)} \Omega_{m} \geq C_{0} r^{2} .
$$

By (25) and (26) we can see $\tilde{u}\left(k_{n_{m}}, S^{1}\right) \cap \operatorname{Int} N_{m}=\varnothing$ and $\tilde{u}\left(k_{n_{m+1}}, S^{1}\right) \cap \operatorname{Int} N_{m}=\varnothing$. This contradicts (32). Thus, $T \neq 0$.

Case 2: $a\left(s_{k}, t_{k}\right) \rightarrow-\infty$.

We deal with this case similarly.

Corollary 3.8. Under the assumptions of Theorem 3.7, there exists a number $T>0$ such that, as $s \rightarrow \pm \infty$,

$$
\partial^{\beta}[a(s, t)-T s] \rightarrow 0
$$

uniformly in $t$, provided $\beta=\left(\beta_{1}, \beta_{2}\right) \in \mathbb{Z}_{\geq 0} \times \mathbb{Z}_{\geq 0}$ and $|\beta|=\beta_{1}+\beta_{2} \geq 1$.

Proof. By Theorem 3.7, there exist a number $T>0$ and a sequence of numbers $s_{k}^{\prime}$ such that $s_{k}^{\prime} \rightarrow+\infty$ and $v\left(s_{k}^{\prime}, \cdot\right) \rightarrow x(T \cdot)$, for some $T$-periodic orbit $x$ of $\boldsymbol{R}_{\infty}$. Suppose (33) is not true for this $T$. Then there exists a sequence of points $\left(s_{k}, t_{k}\right)$ such that $s_{k} \rightarrow+\infty$ and $\left.\partial^{\beta}[a(s, t)-T s]\right|_{\left(s_{k}, t_{k}\right)} \rightarrow c$ as $k \rightarrow+\infty$ for some $|\beta| \geq 1$, where $c$ is a nonzero constant (or $\pm \infty$ ). Define $\bar{a}_{k}(s, t):=a\left(s+s_{k}, t+t_{k}\right)-a\left(s_{k}, t_{k}\right)$ 
and then $\bar{a}_{k}(0,0)=0$. From the proof of Theorem 3.7 we get a subsequence of $k$, still called $k$, and a $T^{\prime}$-periodic orbit $x^{\prime}$ of $\boldsymbol{R}_{\infty}$ such that $\bar{a}_{k} \rightarrow T^{\prime} s$ in $C_{\mathrm{loc}}^{\infty}\left(\mathbb{R}^{+} \times S^{1}, \mathbb{R}\right)$. By a straightforward modification of the proof of Proposition 2.1 in [Hofer et al. 2001] to the Morse-Bott case, we can show that $x^{\prime}$ and $x$ lie in the same component of $N_{T}$ (see Definition 2.7) and in particular $T^{\prime}=T$. Thus,

$$
\begin{aligned}
\left.\partial^{\beta}[a(s, t)-T s]\right|_{\left(s_{k}, t_{k}\right)} & =\left.\partial^{\beta}\left[a\left(s+s_{k}, t+t_{k}\right)-a\left(s_{k}, t_{k}\right)-T s\right]\right|_{(0,0)} \\
& =\left.\partial^{\beta}\left(\bar{a}_{k}(s, t)-T s\right)\right|_{(0,0)} \\
& \rightarrow 0,
\end{aligned}
$$

which contradicts the assumption.

To prove Theorems 2.8 and 2.9, we need to obtain exponential decay estimates.

3C. Exponential decay estimates. In this subsection, we will follow the schemes in [Bourgeois 2002] to prove Theorems 2.8 and 2.9. The strategy is as follows: firstly, we pick a neighborhood $U$ of the orbit $\gamma$, restrict the $J$-holomorphic curve $\tilde{u}$ to a sequence of cylinders inside the domain so that the images lie in the neighborhood and satisfy certain inequalities, and estimate the behaviors of each finite cylinder by the behaviors of boundaries of the cylinder. Secondly, since we have a sequence of circles in the domain whose images lie in $U$, we get that the cylinders bounded by the circles also lie in $U$, based on the estimates. We also show that near the end of the domain $\tilde{u}$ satisfies the inequalities. Once these are achieved, Theorems 2.8 and 2.9 follow easily.

In order to study the $J$-holomorphic curve equation around $\gamma$, we need to introduce a good coordinate chart around a neighborhood of $\gamma$.

Lemma 3.9 [Bourgeois et al. 2003]. Suppose that $J_{\infty}$ is a cylindrical almost complex structure of the Morse-Bott type on $\mathbb{R}^{+} \times V$ at $\infty$. Let $N$ be a component of the set $N_{T} \subset V$ (see Definition 2.7), and let $\gamma$ be one of the orbits from $N$.

(a) If $T$ is the minimal period of $\gamma$ then there exists a neighborhood $U \supset \gamma$ in $V$ such that $U \cap N$ is invariant under the flow of $\boldsymbol{R}_{\infty}$, and one finds coordinates $\left(\vartheta, x_{1}, \ldots, x_{n}, y_{1}, \ldots, y_{n}\right)$ of $U$ such that

$$
N=\left\{x_{1}, \ldots, x_{p}=0, y_{1}, \ldots, y_{q}=0\right\},
$$

for $0 \leq p, q \leq n$, and

$$
\left.\boldsymbol{R}_{\infty}\right|_{N}=\frac{\partial}{\partial \vartheta},\left.\quad \omega_{\infty}\right|_{N}=\left.\omega_{0}\right|_{N}
$$

where $\omega_{0}=\sum_{i=1}^{n} d x_{i} \wedge d y_{i}$.

(b) If $\gamma$ is an m-multiple of a trajectory $\bar{\gamma}$ of a minimal period $T / m$ then there exists a tubular neighborhood $\bar{U}$ of $\bar{\gamma}$ such that its m-multiple cover $U$ together 
with all the structures induced by the covering map from $U \rightarrow \bar{U}$ from the corresponding objects on $\bar{U}$ satisfy the properties of part (a).

Proof. Refer to Lemma A.1 in [Bourgeois et al. 2003].

Using this coordinate chart, we can work locally in $U \subset(\mathbb{R} / T \mathbb{Z}) \times \mathbb{R}^{2 n}$ and make $T$ the minimal period of $\gamma$. Denote by $z_{\text {in }}$ the coordinate $\left(x_{1}, \ldots, x_{p}, y_{1}, \ldots, y_{q}\right)$ and by $z_{\text {out }}$ the coordinate $\left(x_{n-p+1}, \ldots, x_{n}, y_{n-p+1}, \ldots, y_{n}\right)$. We easily obtain the following lemma about the behavior of a $J$-holomorphic curve in the $z_{\text {out }}$ direction.

Lemma 3.10. Let $J$ be an asymptotically cylindrical almost complex structure on $W=\mathbb{R}^{+} \times V$, and let $\tilde{u}$ be a finite Hofer energy J-holomorphic curve from $\mathbb{R}^{+} \times S^{1}$ to $W$. Suppose $\left[m_{k}, n_{k}\right]$ is a sequence of intervals in $\mathbb{R}^{+}$with $m_{k} \rightarrow+\infty$ and $\tilde{u}\left(\left[m_{k}, n_{k}\right] \times S^{1}\right) \subset U$. Then we have, as $k \rightarrow+\infty$,

$$
\sup _{(s, t) \in\left[m_{k}, n_{k}\right] \times S^{1}}\left|\partial^{\beta} z_{\text {out }}(s, t)\right| \rightarrow 0
$$

for all $\beta \in \mathbb{Z}_{\geq 0} \times \mathbb{Z}_{\geq 0}$.

Proof. The proof is very similar to the proof of Corollary 3.8, so we omit it here.

Let's study the $J$-holomorphic curve equation in $\mathbb{R}^{+} \times U \subset \mathbb{R}^{+} \times(\mathbb{R} / T \mathbb{Z}) \times \mathbb{R}^{2 n}$. Define $\theta:=\left[s_{0}, s_{1}\right] \times S^{1}$ for some $s_{0}<s_{1}$ and let $\tilde{u}=(a, \vartheta, z): \theta \rightarrow \mathbb{R} \times U$ be a $J$-holomorphic curve. Then we have

$$
\left(a_{s}, \vartheta_{s}, z_{s}\right)+J(\tilde{u})\left(a_{t}, \vartheta_{t}, z_{t}\right)=0 .
$$

Rewriting this equation according to its $z_{-}^{-}, \vartheta$-, and $a$-components we get ${ }^{3}$

$$
\begin{gathered}
z_{s}+M z_{t}+S z_{\mathrm{out}}+L=0, \\
a_{s}-\vartheta_{t}+B z_{\mathrm{out}}+B^{\prime} z_{t}+N=0, \\
a_{t}+\vartheta_{s}+C z_{\mathrm{out}}+C^{\prime} z_{s}+O=0,
\end{gathered}
$$

where $M, S, B, B^{\prime}, C, C^{\prime}$ depend on $a(s, t), \vartheta(s, t), z(s, t)$ and are bounded by a constant $C_{0}$, and $L, N, O$ depend on $a(s, t), \vartheta(s, t), z(s, t)$ and are bounded by $C_{0} e^{-\delta a}$.

Define an operator $A(s): W^{1,2}\left(S^{1}, \mathbb{R}^{2 n}\right) \rightarrow L^{2}\left(S^{1}, \mathbb{R}^{2 n}\right)$ by

$$
(A(s) w)(t)=-M(\tilde{u}(s, t)) w_{t}(t)-S(\tilde{u}(s, t)) w_{\text {out }}(t) .
$$

Then by (35) we get

$$
A(s) z(s, \cdot)=z_{s}+L
$$

${ }^{3}$ From (35) we can see that if we require $z, z_{s}$ and $z_{t}$ to decay exponentially, $L$ must decay exponentially. The condition $f_{s}^{*} J \rightarrow J_{\infty}$ in $C_{\mathrm{loc}}^{\infty}$ is not enough to guarantee that $L$ decays exponentially. 
Notice that $A(s)$ depends on the map $\tilde{u}=\left(a, \vartheta, z_{\text {in }}, z_{\text {out }}\right)$. If we do not use the original $J$-holomorphic curve $\tilde{u}$ and instead we substitute $\vartheta(s, t)=\vartheta\left(s_{0}, 0\right)+T t$, $a(s, t)=T s, z_{\text {out }}(s, t)=0$, and $z_{\text {in }}(s, t)=z_{\text {in }}\left(s_{0}, t\right)$, then we get another operator denoted by $\tilde{A}(s)$. We can easily see that $\lim _{s \rightarrow+\infty} \tilde{A}(s)$ exists and denote the limiting operator by $A_{0}$. Similarly, we get two matrices $M_{0}(t)$ and $S_{0}(t)$, and then we have

$$
M_{0}(t)^{2}=-\mathrm{id},
$$

and

$$
\left(A_{0} w\right)(t)=-M_{0}(t) w_{t}(t)-S_{0}(t) w_{\text {out }} .
$$

Consider an inner product on $L^{2}\left(S^{1}, \mathbb{R}^{2 n}\right)$ defined by

$$
\langle u, v\rangle_{0}=\int_{0}^{1}\left\langle u,-J_{0} M_{0} v\right\rangle d t,
$$

where the inner product is given by $\langle\cdot, \cdot\rangle=\omega_{0}\left(\cdot, J_{0} \cdot\right)$, and $J_{0}$ is the standard complex structure on $\mathbb{R}^{2 n}$. With respect to the inner product $\langle\cdot, \cdot\rangle_{0}$, one can check directly that $M_{0}$ is antisymmetric and that $A_{0}$ is self-adjoint.

Remark 3.11. $A_{0}$ is injective if and only if $\gamma$ is nondegenerate.

It is not hard to see that ker $A_{0}$ consists of the constant vector fields in $N$ along $\gamma_{0}$. Denote by $P_{0}$ the projection onto ker $A_{0}$ with respect to $\langle\cdot, \cdot\rangle_{0}$, and let $Q_{0}:=I-P_{0}$. It is easy to check the following lemma.

Lemma 3.12. $Q_{0}$ satisfies

$$
\left(Q_{0} w\right)_{t}=w_{t}, \quad\left(Q_{0} w\right)_{s}=Q_{0} w_{s}, \quad\left(Q_{0} w\right)_{\text {out }}=w_{\text {out }}, \quad Q_{0} A_{0}=A_{0} Q_{0} .
$$

The following lemma will be needed in proving Lemma 3.14.

Lemma 3.13. There exists a constant $C>0$ such that

$$
\left\|A_{0} Q_{0} w\right\|_{0} \geq C\left(\left\|Q_{0} w\right\|_{0}+\left\|\left(Q_{0} w\right)_{t}\right\|_{0}\right)
$$

for $w \in W^{1,2}\left(S^{1}, \mathbb{R}^{2 n}\right)$, where $\|\cdot\|_{0}$ is defined using the inner product $\langle\cdot, \cdot\rangle_{0}$.

Proof. To prove the lemma we only need to prove that $\left\|A_{0} Q_{0} w\right\|_{0} \geq C^{\prime}\left\|Q_{0} w\right\|_{0}$ for some $C^{\prime}>0$, because by definition we have

$$
A_{0} Q_{0} w=-M_{0}\left(Q_{0} w\right)_{t}-S_{0} Q_{0} w .
$$

Suppose to the contrary that there exist an $\varepsilon_{n} \rightarrow 0$ and $w_{n} \in W^{1,2}\left(S^{1}, \mathbb{R}^{2 n}\right)$ satisfying $\left\|Q_{0} w_{n}\right\|_{0}=1$ and $\left\|A_{0} Q_{0} w_{n}\right\|_{0} \leq \varepsilon_{n}$. Then we have

$$
\left\|\left(Q_{0} w_{n}\right)_{t}\right\|_{0} \leq\left\|M_{0} A_{0} Q_{0} w_{n}\right\|_{0}+\left\|M_{0} S_{0} Q_{0} w_{n}\right\|_{0} \leq \varepsilon_{n}+C^{\prime \prime} .
$$


Therefore, $Q_{0} w_{n}$ is bounded in $W^{1,2}\left(S^{1}, \mathbb{R}^{2 n}\right)$. Since $W^{1,2}\left(S^{1}, \mathbb{R}^{2 n}\right)$ embeds compactly in $L^{2}\left(S^{1}, \mathbb{R}^{2 n}\right)$ we get a subsequence of $w_{n}$, still denoted by $w_{n}$, such that $Q_{0} w_{n}$ is a Cauchy sequence in $L^{2}\left(S^{1}, \mathbb{R}^{2 n}\right)$. But it is easy to see that $\left(Q_{0} w_{n}\right)_{t}$ is also a Cauchy sequence in $L^{2}\left(S^{1}, \mathbb{R}^{2 n}\right)$. Therefore, $Q_{0} w_{n}$ converges to some $\eta$ in $W^{1,2}\left(S^{1}, \mathbb{R}^{2 n}\right)$, so $\eta$ is an element of ker $A_{0}$. Because $\eta$ also lies in the orthogonal complement of $\operatorname{ker} A_{0}$, we must have $\eta=0$, which contradicts the fact that $\|\eta\|_{0}=\lim _{n \rightarrow 0}\left\|Q_{0} w_{n}\right\|_{0}=1$.

Define $\kappa_{0}(s):=\left(\vartheta\left(s_{0}, 0\right)-\vartheta(s, 0), z_{\text {in }}\left(s_{0}, 0\right)-z_{\text {in }}(s, 0)\right), g_{0}(s):=\frac{1}{2}\left\|Q_{0} z(s)\right\|_{0}^{2}$.

Lemma 3.14. There exist $\delta=\delta(\beta)>0, b=b(\beta)>0$ and $\bar{\kappa}=\bar{\kappa}(\beta)>0$ such that if, for any multi-indices $\beta$,

$$
a\left(s_{0}, 0\right) \geq b, \quad\left|\kappa_{0}\left(s_{0}\right)\right| \leq \bar{\kappa}, \quad \sup _{(s, t) \in \theta}\left|\partial^{\beta} z_{\text {out }}(s, t)\right| \leq \delta,
$$

and, for any multi-indices $\beta$ with $|\beta|>0$,

$$
\sup _{(s, t) \in \theta}\left|\partial^{\beta}(a(s, t)-T s)\right| \leq \delta, \quad \sup _{(s, t) \in \theta}\left|\partial^{\beta}(\vartheta(s, t)-T t)\right| \leq \delta, \quad \sup _{(s, t) \in \theta}\left|\partial^{\beta} z_{\text {in }}(s, t)\right| \leq \delta,
$$

then we have, for $s \in\left[s_{0}, \mathfrak{s}\right]$,

$$
g_{0}^{\prime \prime}(s) \geq c^{2} g_{0}(s)-c_{2} e^{-c_{1}\left(s-s_{0}\right)},
$$

where

$$
\mathfrak{s}:=\sup \left\{s \in\left[s_{0}, s_{1}\right]:\left|\kappa_{0}\left(s^{\prime}\right)\right| \leq \bar{\kappa} \text { for all } s^{\prime} \in\left[s_{0}, s\right]\right\},
$$

and $c, c_{1}, c_{2}>0$ are constants independent of $s_{0}$ and $s_{1}$.

Proof. All constants in the proof may depend on $\beta$. Notice that from the assumption we have

$$
\sup _{(s, t) \in \theta}\left|\partial^{\beta}(\vartheta(s, t)-\vartheta(s, 0)-T t)\right| \leq \delta, \quad \sup _{(s, t) \in \theta}\left|\partial^{\beta}\left(z_{\text {in }}(s, t)-z_{\text {in }}(s, 0)\right)\right| \leq \delta,
$$

for all multi-indices $\beta$.

Define an operator $\bar{A}(s) w=-\bar{M}(\tilde{u}(s, t)) w_{t}(t)-\bar{S}(\tilde{u}(s, t)) w_{\text {out }}(t)$ in the same way as $A(s)$ but using $J_{\infty}$ instead of $J$.

From (38) we get

$$
z_{s}=A_{0} z+\left(\Delta_{0}+\tilde{\Delta}_{0} \kappa_{0}\right) z_{t}+\left(\hat{\Delta}_{0}+\bar{\Delta}_{0} \kappa_{0}\right) z_{\text {out }}+[A(s)-\bar{A}(s)] z-L .
$$

Applying $Q_{0}$ to (42) gives us

$$
\begin{aligned}
\left(Q_{0} z\right)_{s}=A_{0} Q_{0} z & +Q_{0}\left(\Delta_{0}+\tilde{\Delta}_{0} \kappa_{0}\right)\left(Q_{0} z\right)_{t} \\
& +Q_{0}\left(\hat{\Delta}_{0}+\bar{\Delta}_{0} \kappa_{0}\right)\left(Q_{0} z\right)_{\text {out }}+Q_{0}[A(s)-\bar{A}(s)] z-Q_{0} L,
\end{aligned}
$$


where $\Delta_{0}=\bar{M}_{0}-\bar{M}$ and $\hat{\Delta}_{0}=\bar{S}_{0}-\bar{S}$, satisfying for any multi-indices $\beta$

$$
\sup _{(s, t) \in \theta}\left|\partial^{\beta} \Delta_{0}(s, t)\right| \leq C \delta, \quad \sup _{(s, t) \in \theta}\left|\partial^{\beta} \hat{\Delta}_{0}(s, t)\right| \leq C \delta,
$$

and $\tilde{\Delta}_{0} \kappa_{0}=M_{0}-\bar{M}_{0}$ and $\bar{\Delta}_{0} \kappa_{0}=S_{0}-\bar{S}_{0}$, satisfying for any multi-indices $\beta$

$$
\sup _{(s, t) \in \theta}\left|\partial^{\beta} \tilde{\Delta}_{0}(s, t)\right| \leq C, \quad \sup _{(s, t) \in \theta}\left|\partial^{\beta} \bar{\Delta}_{0}(s, t)\right| \leq C .
$$

We can require $0<\delta<T / 2$, and then we get

$$
a(s, t) \geq a\left(s_{0}, 0\right)+\frac{T}{2}\left(s-s_{0}\right)-\delta \geq(b-\delta)+\frac{T}{2}\left(s-s_{0}\right) .
$$

Because $J$ is an asymptotically cylindrical almost complex structure, we get

$$
\left\|Q_{0} L\right\|_{0} \leq c_{0} e^{-c_{0}^{\prime}(b-\delta)} e^{-c_{0}^{\prime} \frac{T}{2}\left(s-s_{0}\right)}
$$

for some constants $c_{0}, c_{0}^{\prime}>0$. Define $c_{1}:=c_{0}^{\prime} T / 2$ and $c_{2}:=c_{0} e^{-c_{0}^{\prime}(b-\delta)}$. Then we have

$$
\left\|Q_{0} L\right\|_{0} \leq c_{2} e^{-c_{1}\left(s-s_{0}\right)} .
$$

We also have

$$
\left\|\left\{\partial^{\beta}[A(s)-\bar{A}(s)]\right\} z\right\|_{0} \leq c_{2} e^{-c_{1}\left(s-s_{0}\right)}\left\|Q_{0} z\right\|_{0, W^{1,2}}
$$

for multi-indices $\beta$, by picking $c_{0}$ larger if necessary.

Now we are ready to estimate $g_{0}^{\prime \prime}(s)$. Obviously we have

$$
g_{0}^{\prime \prime}(s) \geq\left\langle Q_{0} z_{s s}, Q_{0} z\right\rangle_{0} .
$$

Now let's compute the right-hand side of the above inequality. Differentiating (43) with respect to $s$, we obtain

$$
\begin{aligned}
\left(Q_{0} z\right)_{s s}= & A_{0} Q_{0} z_{s}+Q_{0}\left(\Delta_{0}+\tilde{\Delta}_{0} \kappa_{0}\right)\left(Q_{0} z\right)_{s t}+Q_{0}\left(\Delta_{0}+\tilde{\Delta}_{0} \kappa_{0}\right)_{s}\left(Q_{0} z\right)_{t} \\
& +Q_{0}\left(\hat{\Delta}_{0}+\bar{\Delta}_{0} \kappa_{0}\right)\left(Q_{0} z_{s}\right)_{\mathrm{out}}+Q_{0}\left(\hat{\Delta}_{0}+\bar{\Delta}_{0} \kappa_{0}\right)_{s}\left(Q_{0} z\right)_{\mathrm{out}} \\
& +Q_{0}[A(s)-\bar{A}(s)]_{s} z+Q_{0}[A(s)-\bar{A}(s)] z_{s}-Q_{0} L_{s} .
\end{aligned}
$$

Thus we see that $\left\langle Q_{0} z_{s s}, Q_{0} z\right\rangle_{0}$ contains 8 terms. When we are estimating these terms, each time we see $Q_{0} z_{s}$, we replace it using (43). A straightforward calculation using Lemma 3.13 and the fact that

$$
-c_{2} e^{-c_{1}\left(s-s_{0}\right)}\left\|Q_{0} z\right\|_{0, W^{1,2}} \geq-c_{2} e^{-c_{1}\left(s-s_{0}\right)}-c_{2} e^{-c_{1}\left(s-s_{0}\right)}\left\|Q_{0} z\right\|_{0, W^{1,2}}^{2}
$$

gives us

$$
g_{0}^{\prime \prime}(s) \geq\left(1-10 C \delta-10 C\left|\kappa_{0}\right|-10 C c_{2} e^{-c_{1}\left(s-s_{0}\right)}\right) g_{0}(s)-c_{2} e^{-c_{1}\left(s-s_{0}\right)} .
$$


From the definition of $c_{2}$ we can see that if $b$ is large enough, $c_{2}$ can be very close to 0 . Therefore,

$$
g_{0}^{\prime \prime}(s) \geq c^{2} g_{0}(s)-c_{2} e^{-c_{1}\left(s-s_{0}\right)} .
$$

We can require further that $c_{1}>c>0$.

From Lemma 3.14 we easily obtain the following lemma.

Lemma 3.15. Under the same assumption as in Lemma 3.14, we have for $s_{0} \leq s \leq \mathfrak{s}$,

$$
g_{0}(s) \leq \max \left\{g_{0}\left(s_{0}\right), g_{0}(\mathfrak{s})\right\} \frac{\cosh \left[c\left(s-\frac{s_{0}+\mathfrak{s}}{2}\right)\right]}{\cosh \left(c \frac{\mathfrak{s}-s_{0}}{2}\right)}+\frac{c_{2}}{c_{1}^{2}-c^{2}} \frac{\sinh (c(\mathfrak{s}-s))}{\sinh \left(c\left(\mathfrak{s}-s_{0}\right)\right)} .
$$

Proof. Let

$$
\begin{aligned}
h(s):= & \max \left\{g_{0}\left(s_{0}\right), g_{0}(\mathfrak{s})\right\} \frac{\cosh \left[c\left(s-\frac{s_{0}+\mathfrak{s}}{2}\right)\right]}{\cosh \left(c \frac{\mathfrak{s}-s_{0}}{2}\right)}+\frac{c_{2}}{c_{1}^{2}-c^{2}} \frac{1}{\sinh \left(c\left(\mathfrak{s}-s_{0}\right)\right)} \\
& \times\left\{\sinh (c(\mathfrak{s}-s))+e^{-c_{1}\left(\mathfrak{s}-s_{0}\right)} \sinh \left(c\left(s-s_{0}\right)\right)-e^{-c_{1}\left(s-s_{0}\right)} \sinh \left(c\left(\mathfrak{s}-s_{0}\right)\right)\right\} .
\end{aligned}
$$

Then $h(s)$ satisfies

$$
\left\{\begin{array}{l}
h^{\prime \prime}(s)-c^{2} h(s)=-c_{2} e^{-c_{1}\left(s-s_{0}\right)}, \\
h\left(s_{0}\right)=\max \left\{g_{0}\left(s_{0}\right), g_{0}(\mathfrak{s})\right\}, \\
h(\mathfrak{s})=\max \left\{g_{0}\left(s_{0}\right), g_{0}(\mathfrak{s})\right\} .
\end{array}\right.
$$

Let $l(s):=g_{0}(s)-h(s)$. Then $l(s)$ satisfies

$$
\left\{\begin{array}{l}
l^{\prime \prime}(s)-c^{2} l(s) \geq 0 \\
l\left(s_{0}\right) \leq 0 \\
l(\mathfrak{s}) \leq 0
\end{array}\right.
$$

Then by the maximal principle we get $l(s) \leq 0$ for $s_{0} \leq s \leq \mathfrak{s}$. Now the lemma follows from the fact that

$$
e^{-c_{1}\left(\mathfrak{s}-s_{0}\right)} \sinh \left(c\left(s-s_{0}\right)\right)-e^{-c_{1}\left(s-s_{0}\right)} \sinh \left(c\left(\mathfrak{s}-s_{0}\right)\right) \leq 0 .
$$

Now let's study the component $z_{\text {in }}$.

Lemma 3.16. Let e be a unit vector in $\mathbb{R}^{2 n}$ with $e_{\mathrm{out}}=0$. Under the assumption of Lemma 3.14 and for $s \in\left[s_{0}, \mathfrak{s}\right]$, we have

$$
\left|\langle z(s), e\rangle_{0}-\left\langle z\left(s_{0}\right), e\right\rangle_{0}\right| \leq \frac{8 C}{c} \max \left(\left\|Q_{0} z\left(s_{0}\right)\right\|_{0},\left\|Q_{0} z(\mathfrak{s})\right\|_{0}\right)+o\left(c_{2}\right),
$$

where $o\left(c_{2}\right)$ satisfies $\lim _{c_{2} \rightarrow 0} o\left(c_{2}\right)=0$, and $C$ is a constant independent of $s_{0}, s_{1}$. Proof. The inner product of the Cauchy-Riemann equation (35) with $e$ gives

$$
\frac{d}{d s}\langle z, e\rangle_{0}+\left\langle M z_{t}, e\right\rangle_{0}+\left\langle S z_{\text {out }}, e\right\rangle_{0}+\langle L, e\rangle_{0}=0 .
$$


From

$$
\begin{aligned}
\left\langle M z_{t}, e\right\rangle_{0} & =\int_{0}^{1} \omega_{0}\left(M\left(Q_{0} z\right)_{t}, M_{0} e\right) d t \\
& =-\int_{0}^{1} \omega_{0}\left(M_{t} Q_{0} z, M_{0} e\right) d t-\int_{0}^{1} \omega_{0}\left(M Q_{0} z,\left(M_{0}\right)_{t} e\right) d t
\end{aligned}
$$

we can see that

$$
\left|\left\langle M z_{t}, e\right\rangle_{0}\right| \leq C\left\|Q_{0} z\right\|_{0} .
$$

Together with the facts $\left|\left\langle S z_{\text {out }}, e\right\rangle_{0}\right| \leq C\left\|Q_{0} z\right\|_{0}$ and $\left|\langle L, e\rangle_{0}\right| \leq c_{2} e^{-c_{1}\left(s-s_{0}\right)}$ we get

$$
\begin{aligned}
\langle z(s), e\rangle_{0}-\left\langle z\left(s_{0}\right), e\right\rangle_{0} & \leq \int_{s_{0}}^{s}\left[2 C\left\|Q_{0} z(\mathfrak{x})\right\|_{0}+c_{2} e^{-c_{1}\left(\mathfrak{x}-s_{0}\right)}\right] d \mathfrak{x} \\
& \leq 2 C \int_{s_{0}}^{s} \sqrt{2 g_{0}(\mathfrak{x})} d \mathfrak{x}+\frac{c_{2}}{c_{1}} .
\end{aligned}
$$

The proof is finished with a straightforward calculation using Lemma 3.15 and the fact that $\sqrt{\cosh u}<\sqrt{2} \cosh (u / 2)$.

Remark 3.17. By requiring $b$ to be sufficiently large, we can make $c_{2}$ sufficiently small.

Now let's estimate the derivatives of $z$.

Lemma 3.18. There exist $\delta=\delta(\beta)>0, b=b(\beta)>0$ and $\bar{\kappa}=\bar{\kappa}(\beta)>0$ such that if, for any multi-indices $\beta$,

$$
\sup _{(s, t) \in \theta}\left|\partial^{\beta} z_{\text {out }}(s, t)\right| \leq \delta, \quad a\left(s_{0}, 0\right) \geq b,
$$

and, for any multi-indices $\beta$ with $|\beta|>0$,

$$
\sup _{(s, t) \in \theta}\left|\partial^{\beta}(a(s, t)-T s)\right| \leq \delta, \quad \sup _{(s, t) \in \theta}\left|\partial^{\beta}(\vartheta(s, t)-t)\right| \leq \delta, \quad \sup _{(s, t) \in \theta}\left|\partial^{\beta} z_{\text {in }}(s, t)\right| \leq \delta,
$$

then we have, for $s \in\left[s_{0}, \mathfrak{s}\right]$,

$$
\begin{gathered}
\left\|\partial^{\beta} z(s)\right\|_{0} \leq C_{\beta} \max _{\left|\beta^{\prime}\right| \leq|\beta|}\left\{\left\|Q_{0} \partial^{\beta^{\prime}} z\left(s_{0}\right)\right\|_{0},\left\|Q_{0} \partial^{\beta^{\prime}} z(\mathfrak{s})\right\|_{0}\right\} \sqrt{\frac{\cosh \left(c_{1}\left(s-\frac{s_{0}+\mathfrak{s}}{2}\right)\right)}{\cosh \left(c_{1}\left(\frac{s_{0}-\mathfrak{s}}{2}\right)\right)}} \\
+D_{\beta}\left(c_{2}\right) \sqrt{\frac{\sinh (c(\mathfrak{s}-s))}{\sinh \left(c\left(\mathfrak{s}-s_{0}\right)\right)}}+c_{2} e^{-c_{1}\left(s-s_{0}\right)}
\end{gathered}
$$

where

$$
\mathfrak{s}:=\sup \left\{s \in\left[s_{0}, s_{1}\right]:\left|\kappa_{0}\left(s^{\prime}\right)\right| \leq \bar{\kappa} \text { for all } s^{\prime} \in\left[s_{0}, s\right]\right\}
$$


and $C_{\beta}, c_{1}>0$ are constants independent of $s_{0}$ and $s_{1}$, and $D_{\beta}\left(c_{2}\right)$ is a function of $c_{2}$ independent of $s_{0}$ and $s_{1}$, satisfying $\lim _{c_{2} \rightarrow 0} C^{\beta}\left(c_{2}\right)=0$, and $l$ is the integer in Definition 2.1.

Proof. Let's prove the estimate for $|\beta|=1$. The proof of the estimates of the higher derivatives is almost the same. Refer to Lemma A.6 in [Bourgeois et al. 2003] for the estimates for all derivatives in the cylindrical case.

Equation (42) can be rewritten as

$$
z_{s}=A_{0} z+\dot{\Delta} z_{t}+\ddot{\Delta} z_{\text {out }}+\dddot{\Delta} z-L,
$$

with $\dot{\Delta}=\Delta_{0}+\tilde{\Delta}_{0} \kappa_{0}, \ddot{\Delta}=\hat{\Delta}_{0}+\bar{\Delta}_{0} \kappa_{0}$, and $\dddot{\Delta}=[A(s)-\bar{A}(s)]$. If we define $\mathcal{W}:=\left(Q_{0} z, \partial / \partial s\left(Q_{0} z\right), A_{0} Q_{0} z, \partial / \partial s\left(A_{0} Q_{0} z\right)\right)$, then $\mathcal{W}$ satisfies

$$
\mathcal{W}_{s}=\mathcal{A}_{0} \mathcal{W}+\mathcal{Q}_{0} \dot{\Delta} \mathcal{W}_{t}+\mathcal{Q}_{0} \ddot{\Delta} \mathcal{W}_{\text {out }}+\dddot{\Delta} \mathcal{W}-\mathcal{L},
$$

where $\mathcal{A}_{0}=\operatorname{diag}\left(A_{0}, A_{0}, A_{0}, A_{0}\right), \mathcal{Q}_{0}=\operatorname{diag}\left(Q_{0}, Q_{0}, Q_{0}, Q_{0}\right)$, and $\dot{\boldsymbol{\Delta}}, \ddot{\boldsymbol{\Delta}}, \dddot{\boldsymbol{\Delta}}, \mathcal{L}$ satisfy similar estimates as $\dot{\Delta}, \ddot{\Delta}, \dddot{\Delta}, L$ respectively. Indeed, for $|\beta|=1$ we can derive this equation by direct computation. For general $\beta$, we can derive it by induction on $|\beta|$. This equation is of the same type as (47). Copying the proofs of Lemmata 3.14, 3.15 and 3.16, we can get the desired estimate for $\mathcal{W}$. In particular, we get the estimates for $\left(Q_{0} z\right)_{s}$ and $A_{0} Q_{0} z$.

From the equation $z_{t}=M_{0} A_{0} Q_{0} z+M_{0} Q_{0} S_{0} z_{\text {out }}$ we get the estimate for $z_{t}$. Applying $P_{0}$ to (47), we get

$$
\left(P_{0} z\right)_{s}=P_{0} \dot{\Delta} z_{t}+P_{0} \ddot{\Delta} z_{\text {out }}+P_{0} \dddot{\Delta} z-P_{0} L .
$$

This equation together with the estimate of $\dddot{\Delta} z$ (see (44)) gives us the desired estimate for $P_{0} z_{s}$. Then the estimate for $z_{s}$ follows from $z_{s}=P_{0} z_{s}+Q_{0} z_{s}$.

Lemma 3.19. Define

$$
\vartheta_{0}=\int_{0}^{1}\left[\vartheta\left(\frac{s_{0}+\mathfrak{s}}{2}, t\right)-T t\right] d t, \quad a_{0}=\int_{0}^{1}\left[a\left(\frac{s_{0}+\mathfrak{s}}{2}, t\right)-T s_{0}\right] d t,
$$

and define $\tilde{a}=a(s, t)-T s-a_{0}$ and $\tilde{\vartheta}=\vartheta(s, t)-T t-\vartheta_{0}$. Under the assumptions of Lemma 3.18, we have, for $s \in\left[s_{0}, \mathfrak{s}\right]$ and every multi-index $\beta$,

$$
\begin{aligned}
& \| \partial^{\beta}\left(\tilde{a}(s, t)\left\|^{2},\right\| \partial^{\beta}(\tilde{\vartheta}(s, t)) \|^{2}\right. \\
& \quad \leq C_{1} \max _{\left|\beta^{\prime}\right| \leq|\beta|+3}\left\{\left\|Q_{0} \partial^{\beta^{\prime}} z\left(s_{0}\right)\right\|_{0}^{2},\left\|Q_{0} \partial^{\beta^{\prime}} z(\mathfrak{s})\right\|_{0}^{2}\right\} \\
& \quad+C_{1} \max \left\{\left\|\tilde{a}\left(s_{0}, \cdot\right)\right\|^{2}+\left\|\tilde{\vartheta}\left(s_{0}, \cdot\right)\right\|^{2},\|\tilde{a}(\mathfrak{s}, \cdot)\|^{2}+\|\tilde{\vartheta}(\mathfrak{s}, \cdot)\|^{2}\right\}+o\left(c_{2}\right),
\end{aligned}
$$

where $\|\cdot\|$ is the $L^{2}$-norm, o(c $\left.c_{2}\right)$ satisfies $\lim _{c_{2} \rightarrow 0} o\left(c_{2}\right)=0$, and $C_{1}$ is a constant independent of $\tilde{u}$. 
Proof. We can modify the proofs of Lemmata 3.8-3.13 in [Hofer et al. 2002] in the obvious way, similar to what we did in the proof of Lemma 3.14, and then use Lemma 3.18 to prove this lemma. We omit the proof here, since it is essentially not new. ${ }^{4}$

Remark 3.20. When $\mathfrak{s}$ is infinity, we can get a better exponential decay estimate using the same proof, and in that case the term $o\left(c_{2}\right)$ can be replaced by $c_{2} e^{-\left(s-s_{0}\right)}$.

Proof of Theorem 2.8. Let's follow the proof in [Bourgeois 2002]. By Theorem 3.7, we can find a sequence $s_{0 m} \rightarrow \infty$ such that

$$
\lim _{m \rightarrow \infty} u\left(s_{0 m}, t\right)=\gamma(T t), \quad \lim _{m \rightarrow \infty} a\left(s_{0 m}, t\right)= \pm \infty
$$

for some $T$-periodic orbit $\gamma$ of $\boldsymbol{R}_{\infty}$. From the proof of Theorem 3.7, we can further require for any multi-indices $\alpha$ with $|\alpha|>0$ we have $\sup _{t \in S^{1}}\left\|\partial^{\alpha} z\left(s_{0 m}, t\right)\right\| \rightarrow 0$ as $m \rightarrow+\infty$.

Given $\sigma>0$, let $\zeta_{m}>0$ be the largest number such that $u(s, t) \in S^{1} \times[-\sigma, \sigma]^{2 n}$ for all $s \in\left[s_{0 m}, s_{0 m}+\zeta_{m}\right]$. Let $\theta_{m}:=\left[s_{0 m}, s_{0 m}+\zeta_{m}\right] \times S^{1}$ and let $\kappa_{0 m}(s):=$ $\left(\vartheta\left(s_{0 m}, 0\right)-\vartheta(s, 0), z_{\text {in }}\left(s_{0 m}, 0\right)-z_{\text {in }}(s, 0)\right)$. Now we can define the operator $A_{0 m}$, similar to how it was defined before, in the obvious way.

By Corollary 3.8, given $\delta>0$ we have

$$
\sup _{(s, t) \in \theta_{m}}\left|\partial^{\beta}(a(s, t)-T s)\right| \leq \delta
$$

for those multi-indices $\beta$ with $|\beta|>0$, when $m$ is large. This implies that $a\left(s_{0 m}, 0\right) \rightarrow+\infty$ as $m \rightarrow+\infty$. Notice that the other requirements in Lemmata 3.14 and 3.18 are also satisfied; i.e., given $\delta>0$, there exists an $m_{\delta}$ such that for $m>m_{\delta}$ we have

$$
\sup _{(s, t) \in \theta_{m}}\left|\partial^{\beta} z_{\text {out }}(s, t)\right| \leq \delta
$$

for multi-indices $\beta$, and

$$
\begin{gathered}
\sup _{(s, t) \in \theta_{m}}\left|\partial^{\beta}(\vartheta(s, t)-T t)\right| \leq \delta, \\
\sup _{(s, t) \in \theta_{m}}\left|\partial^{\beta} z_{\text {in }}(s, t)\right| \leq \delta
\end{gathered}
$$

for those multi-indices $\beta$ with $|\beta|>0$. Indeed, if $\left\{\left(s_{m_{k}}, t_{m_{k}}\right)\right\}$ violates one of these properties, we can define

$$
\tilde{u}_{m_{k}}(s, t):=\left(a\left(s-s_{m_{k}}, t-t_{m_{k}}\right)-a\left(s_{m_{k}}, t_{m_{k}}\right), u\left(s-s_{m_{k}}, t-t_{m_{k}}\right)\right) .
$$

\footnotetext{
${ }^{4}$ The proof of Proposition 3.4 in [Bourgeois 2002] is inaccurate, and this lemma fills in the gap.
} 
By Ascoli-Arzela, we can extract a subsequence, still called $\tilde{u}_{m_{k}}(s, t)$, such that $\tilde{u}_{m_{k}}(s, t)$ converges in $C_{\text {loc }}^{\infty}$ to a $J_{\infty}$-holomorphic cylinder $\tilde{u}_{\infty}$ over a periodic orbit $\gamma^{\prime} \in N$. Since $\tilde{u}_{\infty}$ must satisfy those three properties, we get a contradiction.

By construction, $\left|\left\langle z\left(s_{0 m}\right), e\right\rangle_{0 m}\right| \rightarrow 0$ and $\left\|Q_{0 m} \partial^{\alpha} z\left(s_{0 m}\right)\right\| \rightarrow 0$, for all multiindices $\alpha$ with $|\alpha| \geq 0$. Let $\bar{\kappa}_{m}$ be the " $\bar{\kappa}$ " in Lemmata 3.14 and 3.18 applied to $\left.\tilde{u}\right|_{\theta_{m}}$ and let $\mathfrak{s}_{m}:=\sup \left\{s \in\left[s_{0 m}, s_{0 m}+\zeta_{m}\right]:\left|\kappa_{0 m}\left(s^{\prime}\right)\right| \leq \bar{\kappa}_{m}\right.$ for all $\left.s^{\prime} \in\left[s_{0}, s\right]\right\}$, and notice that $\bar{\kappa}_{m}$ can actually be chosen independent of $m$. We can extract a subsequence so that $u\left(\mathfrak{s}_{m}, t\right)$ converges to a closed Reeb orbit $\gamma^{\prime \prime} \in N$. Therefore, $\left\|Q_{0 m} \partial^{\alpha} z\left(\mathfrak{s}_{m}\right)\right\| \rightarrow 0$, for all multi-indices $\alpha$ with $|\alpha| \geq 0$. Since $\left\langle z\left(\mathfrak{s}_{m}\right), e\right\rangle_{0} \rightarrow 0$ and $\sup _{t \in S^{1}}\left|(\partial / \partial t) z_{\text {in }}\left(\mathfrak{s}_{m}, t\right)\right| \rightarrow 0$, we obtain $\sup _{t \in S^{1}}\left|z_{\text {in }}\left(\mathfrak{s}_{m}, t\right)\right| \rightarrow 0$. By Lemmata 3.14 and 3.18 , we have

$$
\sup _{s \in\left[s_{0 m}, \mathfrak{s}_{m}\right]}\left\|\partial^{\beta} z(s)\right\|_{0 m} \rightarrow 0
$$

for $|\beta| \leq k$. Therefore,

$$
\begin{aligned}
\sup _{(s, t) \in\left[s_{0 m}, \mathfrak{s}_{m}\right] \times S^{1}}\left|z_{\text {in }}(s, t)\right| & \leq \sup _{s \in\left[s_{0 m}, \mathfrak{s}_{m}\right]}\left\|z_{\text {in }}(s, \cdot)\right\|_{C^{0}\left(S^{1}\right)} \\
& \leq C \sup _{s \in\left[s_{0 m}, \mathfrak{s}_{m}\right]}\left\|z_{\text {in }}(s, \cdot)\right\|_{W^{1,2}\left(S^{1}\right)} \\
& \leq C_{1}\left\{\sup _{s \in\left[s_{0 m}, \mathfrak{s}_{m}\right]}\left\|(\partial / \partial t) z_{\text {in }}(s, \cdot)\right\|_{0 l}+\sup _{s \in\left[s_{0 m}, \mathfrak{s}_{m}\right]}\left\|z_{\text {in }}(s, \cdot)\right\|_{0 m}\right\} \\
& \rightarrow 0 .
\end{aligned}
$$

Lemma 3.19 and formula (48) imply that $\left|\vartheta\left(\mathfrak{s}_{m}, 0\right)-\vartheta\left(s_{0 m}, 0\right)\right| \rightarrow 0$ as $m \rightarrow \infty$. Thus, we have $\mathfrak{s}_{m}=s_{0 m}+\zeta_{m}$ for $m$ large enough, and

$$
\sup _{(s, t) \in\left[s_{0 m}, s_{0 m}+\zeta_{m}\right] \times S^{1}}|z(s, t)| \rightarrow 0
$$

as $m \rightarrow \infty$. Therefore, $\zeta_{m}=+\infty$ for $m$ large.

Furthermore, we can show that the convergence of a $J$-holomorphic curve is exponentially fast.

Proof of Theorem 2.9. Now with the help of the previous lemmata, the proof of the third inequality is almost evident. Indeed, since $\mathfrak{s}=+\infty$, Lemma 3.15 becomes

$$
g_{0}(s) \leq\left(g_{0}\left(s_{0}\right)+\frac{c_{2}}{c_{1}^{2}-c^{2}}\right) e^{-c\left(s-s_{0}\right)} .
$$

Consequently, in the proof Lemma 3.16, we can get

$$
\left|\langle z(s), e\rangle_{0}\right| \leq \int_{s}^{+\infty}\left[2 C\left\|Q_{0} z(\mathfrak{x})\right\|_{0}+c_{2} e^{-c_{1}\left(\mathfrak{x}-s_{0}\right)}\right] d \mathfrak{x} \leq C^{\prime} e^{-c\left(s-s_{0}\right)},
$$


where $C^{\prime}$ is independent of $s$. Similarly, we can get the corresponding statement of Lemma 3.18 for $\mathfrak{s}=+\infty$.

The proof for the rest is a straightforward modification of the original proof in [Hofer et al. 2001].

So far we have studied the behaviors of a finite energy $J$-holomorphic curve whose domain is an infinite cylinder. In order to compactify the moduli space of holomorphic curves, we also need to understand the behavior of a finite energy $J$-holomorphic curve whose domain is a long but finite interval and whose $\omega$-energy is small. To do that, we need the following lemma.

Lemma 3.21 (bubbling lemma [Bourgeois et al. 2003; Hofer and Viterbo 1992]). Let $J^{0}$ be a cylindrical almost complex structure on $W=\mathbb{R}^{+} \times V$. There exists a constant $\hbar>0$ depending only on $\left(W, J^{0}, \omega^{0}\right)$ where $J^{0}=J_{\infty}^{0}$ and $\omega^{0}=\omega_{\infty}^{0}$ (see Definitions 2.1 and 2.3 and Section $2 B$ ), such that the following holds true. Let $\left(J^{n}, \omega_{\infty}^{n}\right)$ be a sequence of pairs satisfying (AC1)-(AC5) on $W$ and converging to $\left(J^{0}, \omega^{0}\right)$ in the $C_{\mathrm{loc}}^{\infty}$ sense. Consider a sequence of $J^{n}$-holomorphic maps $\tilde{u}_{n}=$ $\left(a_{n}, u_{n}\right)$ from the unit disc $B(0,1)$ to W satisfying $E_{n}\left(\tilde{u}_{n}\right)=E_{\omega^{n}}\left(\tilde{u}_{n}\right)+E_{\lambda_{n}}\left(\tilde{u}_{n}\right) \leq C$ (see Section $2 B$ ) for some constant $C$, such that the sequence $a_{n}(0)$ is bounded, and such that $\left\|\nabla \tilde{u}_{n}(0)\right\| \rightarrow+\infty$ as $n \rightarrow+\infty$. Then there exists a sequence of points $z_{n} \in B(0,1)$ converging to 0 , and sequences of positive numbers $\varepsilon_{n}$ and $R_{n}$ satisfying

$$
\varepsilon_{n} \rightarrow 0, \quad R_{n} \rightarrow+\infty, \quad \varepsilon_{n} R_{n} \rightarrow+\infty, \quad\left|z_{n}\right|+\varepsilon_{n}<1,
$$

such that the rescaled maps

$$
\tilde{u}_{n}^{0}: B\left(0, \varepsilon_{n} R_{n}\right) \rightarrow W, \quad z \mapsto \tilde{u}_{n}\left(z_{n}+R_{n}^{-1} z\right)
$$

converge in $C_{\mathrm{loc}}^{1}$ to a $J_{0}$-holomorphic map $\tilde{u}^{0}: \mathbb{C} \rightarrow W$ which satisfies $E\left(\tilde{u}^{0}\right) \leq C$ and $E_{\omega^{0}}\left(\tilde{u}^{0}\right)>\hbar$.

Moreover, this map is either a $J_{0}$-holomorphic plane asymptotic as $|z| \rightarrow \infty$ to a periodic orbit of the vector field $\boldsymbol{R}^{0}$ defined by $\boldsymbol{R}^{0}=J_{0}(\partial / \partial r)$, or extendable to a $J_{0}$-holomorphic sphere $\mathbb{P}^{1} \rightarrow W$ by Gromov's removal of singularity theorem.

A similar statement is also true for $\mathbb{R}^{-} \times V$.

Proof. See [Hofer and Viterbo 1992].

The following theorem studies the behavior of a long cylinder having small $\omega$-area. It is needed in order to prove the compactness results for the moduli space of $J$-holomorphic curves in symplectic field theory. Refer to [Hofer et al. 2002; Bourgeois et al. 2003] for the cylindrical case.

Theorem 3.22. Suppose that $J$ is an asymptotically cylindrical almost complex structure on $W=\mathbb{R}^{ \pm} \times V$ at $\pm \infty$, and suppose that $J$ is of the Morse-Bott type. 
Given $E_{0}>0$ and $\varepsilon>0$, there exist constants $\sigma, c>0$ such that for every $R>c$ and every J-holomorphic cylinder $\tilde{u}=(a, u):[-R, R] \times S^{1} \rightarrow W$ satisfying the inequalities $E_{\omega}(\tilde{u})<\sigma$ and $E(\tilde{u})<E_{0}$, we have $u(s, t) \in B_{\varepsilon}(u(0, t))$ for all $s \in[-R+c, R-c]$ and all $t \in S^{1}$.

Proof. The proof follows the scheme in [Bourgeois et al. 2003] with some modification.

By contradiction, assume that there exist sequences $c_{n} \rightarrow+\infty, R_{n}>c_{n}$ and $\tilde{u}_{n}=\left(a_{n}, u_{n}\right):\left[-R_{n}, R_{n}\right] \times S^{1} \rightarrow W$. The sequence $\tilde{u}_{n}$ is $J$-holomorphic, satisfying $E\left(\tilde{u}_{n}\right) \leq E_{0}, E_{\omega}\left(\tilde{u}_{n}\right) \rightarrow 0$, and $u_{n}\left(s_{n}, t_{n}\right) \notin B\left(u_{n}\left(0, t_{n}\right), \varepsilon\right)$ for some $s_{n} \in\left[-k_{n}, k_{n}\right]$, $k_{n}=R_{n}-c_{n}$ and $t_{n} \in S^{1}$. By the proof of Proposition 3.4 together with the bubbling lemma (Lemma 3.21), $\left\|\nabla \tilde{u}_{n}\right\|$ is uniformly bounded on each compact subset. We can extract a subsequence of $n$, still denoted by $n$, such that $a_{n}\left(s_{n}, t_{n}\right) \rightarrow \pm \infty$. This is because, otherwise, we can get a contradiction as in the proof of Proposition 3.4. Now define $\tilde{u}_{n}^{0}(s, t):=\left(a_{n}^{0}, u_{n}^{0}\right)=\left(a_{n}(s, t)-a_{n}\left(s_{n}, t_{n}\right), u_{n}(s, t)\right)$. By Ascoli-Arzela, we can extract a subsequence, still called $\tilde{u}_{n}^{0}$, converging to a $J_{\infty}$-holomorphic cylinder $\tilde{u}: \mathbb{R} \times S^{1} \rightarrow \mathbb{R} \times V$. Since $\tilde{u}$ satisfies $E_{\omega}(\tilde{u})=0$ and $E(\tilde{u}) \leq E_{0}$, we have that $\tilde{u}$ is a trivial cylinder over some periodic orbit $\gamma$. Let's choose a neighborhood around $\gamma$, and pick the coordinate as in Lemma 3.9, and show that

$$
\sup _{(s, t) \in\left[-k_{n}, k_{n}\right] \times S^{1}}\left|\partial^{\beta} z_{\text {out }, n}(s, t)\right| \rightarrow 0
$$

for multi-indices $\beta$ and

$$
\begin{gathered}
\sup _{(s, t) \in\left[-k_{n}, k_{n}\right] \times S^{1}}\left|\partial^{\beta}\left(a_{n}(s, t)-T s\right)\right| \rightarrow 0, \\
\sup _{(s, t) \in\left[-k_{n}, k_{n}\right] \times S^{1}}\left|\partial^{\beta} z_{\text {in }, n}(s, t)\right| \rightarrow 0, \\
\sup _{(s, t) \in\left[-k_{n}, k_{n}\right] \times S^{1}}\left|\partial^{\beta}\left(\vartheta_{n}(s, t)-T t\right)\right| \rightarrow 0
\end{gathered}
$$

for multi-indices $\beta$ with $|\beta|>0$, when $n \rightarrow+\infty$.

If this were not true, suppose there exists a subsequence of $\{n\}$, still denoted by $\{n\}$, such that $\left(s_{n}^{\prime}, t_{n}^{\prime}\right)$ violates one of these properties. Then we can make the same argument using $\left(s_{n}^{\prime}, t_{n}^{\prime}\right)$ instead of $\left(s_{n}, t_{n}\right)$ as above and get a trivial cylinder contradicting the fact that $\left(s_{n}^{\prime}, t_{n}^{\prime}\right)$ violates one of these properties.

Define $A_{0 n}$ and $Q_{0 n}$ in the obvious way using $\gamma$ and $s_{0 n}=0$. Then apply Lemmata 3.14-3.16 and 3.18 to each $\left.\tilde{u}_{n}\right|_{\left[-k_{n}, k_{n}\right]}$ to get $\sup _{s \in\left[-k_{n}, k_{n}\right]}\left\|Q_{0 n} z_{n}(s)\right\|_{0, n} \rightarrow 0$. Then the Sobolev embedding theorem tells us that $\kappa_{0 n} \rightarrow 0$ as $n \rightarrow+\infty$. This contradicts the assumption that $u_{n}\left(s_{n}, t_{n}\right) \notin B\left(u_{n}(0, t), \varepsilon\right)$.

We need the following theorem later to prove the surjectivity of the gluing map in the subsequent paper. After proving all the previous lemmata and theorems, the 
proof of the following theorem is standard. For the case when $J$ is cylindrical and nondegenerate and $V$ is a contact manifold, the proof is given in [Hofer et al. 2002].

Theorem 3.23. Suppose that $J$ is an asymptotically cylindrical almost complex structure on $W=\mathbb{R}^{+} \times V$ at $\infty$, and suppose that $J$ is of the Morse-Bott type. Given $E_{0}>0$ and sufficiently small $\varepsilon>0$, there exist constants $\sigma, c, b, v>0$ such that, for every $R>c$ and every J-holomorphic cylinder $\tilde{u}=(a, u):[-R, R] \times S^{1} \rightarrow$ $(b, \infty) \times V$ satisfying the inequalities $E_{\omega}(\tilde{u})<\sigma$ and $E(\tilde{u})<E_{0}$, there exists either a point $w \in W$ such that $\tilde{u}(s, t) \in B_{\varepsilon}(w)$ for $s \in[-R+c, R-c]$ and $t \in S^{1}$,or a T-periodic orbit $\gamma$ of $\boldsymbol{R}_{\infty}$ such that $u(s, t) \in B_{\varepsilon}(\gamma(T t))$ for $s \in[-R+c, R-c]$ and $t \in S^{1}$. In the second case, we have a coordinate around $\gamma$ as in Lemma 3.9 such that

$$
\begin{aligned}
\left|D^{\beta}\left\{a(s, t)-T s-a_{0}\right\}\right|^{2} & \leq \varepsilon^{2} M_{\beta} \frac{\cosh (2 v s)}{\cosh (2 v(R-c))}+C_{\beta} e^{-c_{\beta}(s+R-c)}, \\
\left|D^{\beta}\left\{\vartheta(s, t)-T t-\vartheta_{0}\right\}\right|^{2} & \leq \varepsilon^{2} M_{\beta} \frac{\cosh (2 v s)}{\cosh (2 v(R-c))}+C_{\beta} e^{-c_{\beta}(s+R-c)}, \\
\left|D^{\beta} z(s, t)\right|^{2} & \leq \varepsilon^{2} M_{\beta} \frac{\cosh (2 v s)}{\cosh (2 v(R-c))}+C_{\beta} e^{-c_{\beta}(s+R-c)},
\end{aligned}
$$

for $s \in[-R+c, R-c], t \in S^{1}$, and $\beta \in \mathbb{N} \times \mathbb{N}$ such that $|\beta| \leq l-3$, where $M_{\beta}$, $C_{\beta}, c_{\beta}$ are constants independent of $\tilde{u}$ and $\varepsilon$, and $C_{\beta}$ converges to 0 as b converges to $+\infty$, and $M_{\beta}$ and $c_{\beta}$ are independent of $b$.

A similar statement is also true for $\mathbb{R}^{-} \times V$.

\section{Almost complex manifolds with asymptotically cylindrical ends}

In this section, we introduce the notion of almost complex manifolds with asymptotically cylindrical ends.

4A. Definitions. Let $\left(W_{0}, \omega^{\prime}\right)$ be a closed symplectic manifold with boundary $\partial W_{0}=V_{+} \sqcup V_{-}$, where $V_{ \pm}$is an oriented closed manifold. Let $W$ be the noncompact smooth manifold obtained by attaching $E_{ \pm}:=\mathbb{R}^{ \pm} \times V_{ \pm}$to $W_{0}$ along $\{0\} \times V_{ \pm}$and $V_{ \pm}$. Suppose that there exists an almost complex structure $J$ on $W$ such that $\left.J\right|_{W_{0}}$ is compatible with $\omega^{\prime}$ and $\left(E_{ \pm},\left.J\right|_{E_{ \pm}}\right)$is asymptotically cylindrical at $\pm \infty$. We assume that the orientation of $E_{ \pm}$determined by $\left.J\right|_{E_{ \pm}}$coincides with the orientation coming from the standard orientation of $\mathbb{R}^{ \pm}$and the orientation of $V_{ \pm}$. This assumption distinguishes $V_{+}$from $V_{-}$. Furthermore, we assume $\left.\omega^{\prime}\right|_{V_{ \pm}}=\omega_{ \pm \infty}$, where $\omega_{ \pm \infty}$ is the 2-form on $V_{ \pm}$from Definition 2.1. In this case, we say $(W, J)$ is an almost complex manifold with asymptotically cylindrical ends.

Example 4.1 [Bourgeois et al. 2003]. Let $\left(X, \omega^{\prime}, J\right)$ be an almost Kähler manifold, and let $Y \subset X$ be an embedded closed almost Kähler submanifold. We claim 
that $\left(X \backslash Y,\left.J\right|_{X \backslash Y}\right.$ ) has an asymptotically cylindrical negative end. Let $N$ be the normal bundle of $Y$ in $X$ with the metric $\left.\omega^{\prime}(\cdot, J \cdot)\right|_{Y}$, let $V$ be the associated unit sphere bundle of $N$ defined by $V=\{u \in N:|u|=1\}$, and let $U_{\varepsilon}$ be the disc bundle of $N$ defined by $U_{\varepsilon}=\{u \in N:|u| \leq \varepsilon\}$. For small enough $\varepsilon>0$, we have that $U_{\varepsilon}$ is diffeomorphic to a tubular neighborhood of $Y$ in $X$ via the exponential map with respect to the metric $\omega^{\prime}(\cdot, J \cdot)$. Since $U_{\varepsilon} \backslash Y$ is also diffeomorphic to $(-\infty, \log \varepsilon] \times V$ via the map $u \mapsto(\log |u|, u /|u|)$, one can check that this makes $\left(X \backslash Y,\left.J\right|_{X \backslash Y}\right)$ an almost complex manifold with an asymptotically cylindrical negative end.

In particular, if we pick $Y$ to be a point in $X$, we get Example 2.5 as a special case.

4B. Energy of J-holomorphic curves. Let $w$ be a J-holomorphic map from a punctured Riemann surface $(\Sigma, j)$ to $(W, J)$, and define

$$
\begin{aligned}
E_{\text {symp }}(w) & =\int_{w^{-1}\left(W_{0}\right)} w^{*} \omega^{\prime}, \\
E_{\omega}(w) & =\int_{w^{-1}\left(E_{+}\right)} w^{*} \omega+\int_{w^{-1}\left(E_{-}\right)} w^{*} \omega, \\
E_{\lambda}(w) & =\sup _{\phi \in \mathcal{C}_{+}} \int_{w^{-1}\left(E_{+}\right)} w^{*}(\phi \sigma \wedge \lambda)+\sup _{\phi \in \mathcal{C}_{-}} \int_{w^{-1}\left(E_{-}\right)} w^{*}(\phi \sigma \wedge \lambda),
\end{aligned}
$$

where

$\mathcal{C}_{+}=\left\{\phi \in C_{c}^{\infty}\left(\mathbb{R}^{+},[0,1]\right): \int \phi=1\right\}, \quad \mathcal{C}_{-}=\left\{\phi \in C_{c}^{\infty}\left(\mathbb{R}^{-},[0,1]\right): \int \phi=1\right\}$, and

$$
E(w)=E_{\text {symp }}(w)+E_{\omega}(w)+E_{\lambda}(w) .
$$

Theorem 4.2. Suppose $(W, J)$ is an almost complex manifold with asymptotically cylindrical ends, and suppose that $J$ is of the Morse-Bott type. Let $w$ be a $J$-holomorphic curve from a punctured Riemann surface $\Sigma$ to $W$ with $E(w)<\infty$. Then around each puncture, either $w$ can be extended holomorphically over the puncture, or one can choose a holomorphic coordinate chart $\mathbb{R}^{+} \times S^{1}$ or $\mathbb{R}^{-} \times S^{1}$ in $S$ around the puncture such that $w$ converges to a Reeb orbit in $E_{+}$or $E_{-}$in the sense of Theorems 2.8 and 2.9.

Proof. If $w$ is bounded around a puncture, then Gromov's removal of singularity theorem implies that $w$ can be extended holomorphically over the puncture.

Suppose that $w$ is not bounded around a puncture. We pick a holomorphic cylindrical coordinate $\mathbb{R}^{+} \times S^{1}$ around the puncture of $\Sigma$. By Proposition 3.5, 
$|\nabla w|<C$ with respect to the standard metric on $\mathbb{R}^{+} \times S^{1}$. If $w$ keeps coming back to a compact region of $W$ and also escaping to the positive (or negative) end of $W$, we can find an $r_{0}$ such that $w$ touches $\left\{r_{0}\right\} \times V_{ \pm}$and $\left\{r_{0} \pm 3 C\right\} \times V_{ \pm}$ infinitely many times. Then we can apply Gromov's monotonicity theorem to $w$ in the region $\left[r_{0} \pm C, r_{0} \pm 2 C\right] \times V_{ \pm}$as in the argument of Case 1 in the proof of Theorem 3.7, and get $E(w)=\infty$, which contradicts the assumption. Therefore, near the puncture, $w$ converges to $\infty$ or $-\infty$ in $E_{+}$or $E_{-}$. Then Theorem 4.2 follows from Theorems 2.8 and 2.9.

Proposition 4.3. Suppose $(W, J)$ is an almost complex manifold with asymptotically cylindrical ends, and suppose that $J$ is of the Morse-Bott type. Then there exists a constant $\varepsilon_{0}>0$ such that if $K_{0}^{ \pm}<\varepsilon_{0}$, where $K_{0}^{ \pm}$is the constant in (AC1), the following holds.

Let $w$ be a J-holomorphic curve from a punctured Riemann surface $\Sigma$ to $W$ such that, around punctures of $\Sigma$, we have that $w$ converges to the periodic orbits $\gamma_{1}^{+}, \ldots, \gamma_{p}^{+}$inside $V_{+}$and $\gamma_{1}^{-}, \ldots, \gamma_{q}^{-}$inside $V_{-}$. Then

$$
E(w) \leq C_{1} \sum_{i=1}^{p} \int_{\gamma_{i}^{+}} \lambda_{\infty}-C_{2} \sum_{j=1}^{q} \int_{\gamma_{j}^{-}} \lambda_{-\infty}+C_{3}\left\{\int_{w^{-1}\left(E_{+}\right)} w^{*} \omega_{\infty}+\int_{w^{-1}\left(W_{0}\right)} w^{*} \omega^{\prime}+\int_{w^{-1}\left(E_{-}\right)} w^{*} \omega_{-\infty}\right\},
$$

where $C_{1}, C_{2}, C_{3}$ are positive constants that are independent of $w$. In particular, $E(w)$ only depends on the homology class of $w$ in $H_{2}\left(W,\left(\bigcup_{i=1}^{p} \gamma_{i}^{+}\right) \cup\left(\bigcup_{j=1}^{q} \gamma_{j}^{-}\right)\right)$.

Proposition 4.3 is the asymptotically cylindrical version of Proposition 6.13 in [Bourgeois et al. 2003]. The extra work to prove it for the asymptotically cylindrical case is essentially carried out in the Appendix of [Bao 2014] where we assume $\omega_{ \pm \infty}=d \lambda_{\infty}$. For the sake of completeness, we reproduce the proof here.

Proof. First, we restrict ourselves to $E_{+}$and denote $w_{ \pm}:=\left.w\right|_{w^{-1}\left(E_{ \pm}\right)}$. Note that, when restricted to $J$-complex planes, we have

$$
\begin{gathered}
\left|\omega-\omega_{\infty}\right| \leq \varepsilon e^{-\delta s}(\omega+\sigma \wedge \lambda), \\
\left|d \lambda_{\infty}\right| \leq C \omega+\varepsilon e^{-\delta s} \sigma \wedge \lambda, \\
\left|\sigma \wedge \lambda-d r \wedge \lambda_{\infty}\right| \leq \varepsilon e^{-\delta s}(\sigma \wedge \lambda+\omega),
\end{gathered}
$$

where $C$ is a positive constant and the constant $\varepsilon>0$ can be chosen to be small if $K_{0}^{+}$is small. Since $\int_{0}^{\infty} \delta e^{-\delta s} d s=1$, we get

$$
\int_{w^{-1}\left(E_{+}\right)} w^{*} \omega \leq \int_{w^{-1}\left(E_{+}\right)} w^{*} \omega_{\infty}+\varepsilon \int_{w^{-1}\left(E_{+}\right)} w^{*} \omega+\frac{\varepsilon}{\delta} E_{\lambda}\left(w_{+}\right),
$$


where

$$
E_{\lambda}\left(w_{ \pm}\right):=\sup _{\phi \in \mathcal{C}_{ \pm}} \int_{w^{-1}\left(E_{ \pm}\right)} w^{*}(\phi \sigma \wedge \lambda) .
$$

Absorbing the second term on the right-hand side to the left-hand side, we get

$$
E_{\omega}\left(w_{+}\right) \leq C_{1} \int_{w^{-1}\left(E_{+}\right)} w^{*} \omega_{\infty}+C_{2} \varepsilon E_{\lambda}\left(w_{+}\right),
$$

for some constants $C_{1}, C_{2}$, where $E_{\omega}\left(w_{ \pm}\right):=\int_{w^{-1}\left(E_{ \pm}\right)} w^{*} \omega$.

For any $\phi \in \mathcal{C}_{+}$, let $\Phi(s)=\int_{0}^{s} \phi(l) d l$. Then using (55) and (56) we have

$$
\begin{aligned}
\int_{w^{-1}\left(E_{+}\right)} & w^{*} \phi \sigma \wedge \lambda \\
= & \int_{w^{-1}\left(E_{+}\right)} w^{*} \phi d r \wedge \lambda_{\infty}+\int_{w^{-1}\left(E_{+}\right)} w^{*} \phi\left(\sigma \wedge \lambda-d r \wedge d \lambda_{\infty}\right) \\
\leq & \int_{w^{-1}\left(E_{+}\right)} w^{*} d\left(\Phi \lambda_{\infty}\right)-\int_{w^{-1}\left(E_{+}\right)} w^{*} \Phi d \lambda_{\infty}+\int_{w^{-1}\left(E_{+}\right)} w^{*} \varepsilon e^{-\delta s} \phi(\sigma \wedge \lambda+\omega) \\
\leq & \sum_{i=1}^{p} \int_{\gamma_{i}^{+}} \lambda_{\infty}+\int_{w^{-1}\left(E_{+}\right)} w^{*}\left(C \omega+\varepsilon e^{-\delta s} \sigma \wedge \lambda\right)+\int_{w^{-1}\left(E_{+}\right)} w^{*} \varepsilon e^{-\delta s} \phi(\sigma \wedge \lambda+\omega) \\
\leq & \sum_{i=1}^{p} \int_{\gamma_{i}^{+}} \lambda_{\infty}+C E_{\omega}\left(w_{+}\right)+\varepsilon E_{\lambda}\left(w_{+}\right),
\end{aligned}
$$

where in the last inequality we get the constants $C$ and $\varepsilon$ by slightly abusing the notations, but we can still have $\varepsilon$ small. Taking the sup over $\phi$, we get

$$
E_{\lambda}\left(w_{+}\right) \leq \sum_{i=1}^{p} \int_{\gamma_{i}^{+}} \lambda_{\infty}+C E_{\omega}\left(w_{+}\right)+\varepsilon E_{\lambda}\left(w_{+}\right) .
$$

Therefore, by (57) and (58) we have

$$
E_{\omega}\left(w_{+}\right)+E_{\lambda}\left(w_{+}\right) \leq C_{1} \int_{\gamma_{+}} \lambda_{\infty}+C_{2} \int_{w^{-1}\left(E_{+}\right)} w^{*} \omega_{\infty},
$$

where constants $C_{1}$ and $C_{2}$ are not necessarily the same as before. 
For $E_{-}$, by the proof of Theorem 10 in [Bao 2014], if $K_{0}^{-}$is small we have

$$
E_{\omega}\left(w_{-}\right)+E_{\lambda}\left(w_{-}\right) \leq C_{1}^{\prime} E_{\text {symp }}(w)+C_{2}^{\prime} \int_{w^{-1}\left(E_{-}\right)} w^{*} \omega_{\infty}-C_{3}^{\prime} \sum_{j=1}^{q} \int_{\gamma_{j}^{-}} \lambda_{-\infty},
$$

where $C_{1}^{\prime}, C_{2}^{\prime}, C_{3}^{\prime}$ are positive constants independent of $w$. Here we recall that

$$
E_{\text {symp }}(w)=\int_{w^{-1}\left(W_{0}\right)} w^{*} \omega^{\prime}
$$

Now by (59) and (60) we have

$$
\begin{aligned}
E(w) & =E_{\omega}\left(w_{+}\right)+E_{\lambda}\left(w_{+}\right)+E_{\omega}\left(w_{-}\right)+E_{\lambda}\left(w_{+}\right)+E_{\mathrm{symp}}(w) \\
& \leq a_{1}\left(E_{\omega}\left(w_{+}\right)+E_{\lambda}\left(w_{+}\right)\right)+a_{2}\left(E_{\omega}\left(w_{-}\right)+E_{\lambda}\left(w_{+}\right)\right)+a_{3} E_{\mathrm{symp}}\left(w_{0}\right) \\
& \leq C_{1} \int_{\gamma_{+}} \lambda_{\infty}-C_{2} \int_{\gamma_{-}} \lambda_{-\infty}+C_{3}\left\{\int_{w^{-1}\left(E_{+}\right)} w^{*} \omega_{\infty}+\int_{w^{-1}\left(W_{0}\right)} w^{*} \omega^{\prime}+\int_{w^{-1}\left(E_{-}\right)} w^{*} \omega_{\infty}\right\},
\end{aligned}
$$

where $a_{1}, a_{2}, a_{3} \geq 1$ are positive constants chosen in a way such that the last inequality holds for some positive constants $C_{1}, C_{2}$ and $C_{3}$.

Let $\mathcal{M}_{g, p+q}^{A}\left(\gamma_{1}^{+}, \ldots, \gamma_{p}^{+}, \gamma_{1}^{-}, \ldots, \gamma_{q}^{-} ; J\right)$ be the moduli space of $J$-holomorphic curves of genus $g$ in $W$ that converge to periodic orbits $\gamma_{1}^{+}, \ldots, \gamma_{p}^{+}$inside $V_{+}$and $\gamma_{1}^{-}, \ldots, \gamma_{q}^{-}$inside $V_{-}$and represent the homology class $A$, which is an element of $H_{2}\left(W,\left(\bigcup_{i=1}^{p} \gamma_{i}^{+}\right) \cup\left(\bigcup_{j=1}^{q} \gamma_{j}^{-}\right)\right)$. Let $\overline{\mathcal{M}}_{g, p+q}^{A}\left(\gamma_{1}^{+}, \ldots, \gamma_{p}^{+}, \gamma_{1}^{-}, \ldots, \gamma_{q}^{-} ; J\right)$ be the compactification of the space $\mathcal{M}_{g, p+q}^{A}\left(\gamma_{1}^{+}, \ldots, \gamma_{p}^{+}, \gamma_{1}^{-}, \ldots, \gamma_{q}^{-} ; J\right)$ by allowing stable holomorphic buildings. See Theorems 8.1 and 8.2 in [Bourgeois et al. 2003] for the definition of stable holomorphic buildings in manifolds with cylindrical ends and the topology of the moduli space of holomorphic buildings. Finally, let us state the compactness results.

Theorem 4.4. Suppose $(W, J)$ is an almost complex manifold with asymptotically cylindrical ends, and suppose that $J$ is of the Morse-Bott type. Then $\overline{\mathcal{M}}_{g, p+q}^{A}\left(\gamma_{1}^{+}, \ldots, \gamma_{p}^{+}, \gamma_{1}^{-}, \ldots, \gamma_{q}^{-} ; J\right)$ is compact.

Proof. The extra difficulty of proof that comes from $J$ being asymptotically cylindrical is taken care of by Theorem 4.2; the rest of the proof is a straightforward modification of [Bourgeois et al. 2003]. For the sake of completeness, we outline the proof as follows.

Suppose that $\left(\Sigma_{n}, w_{n}\right)$ is a sequence of $J$-holomorphic maps from a punctured Riemann surface $\Sigma_{n}$, with $E\left(w_{n}\right)<C$.

First, we add additional marked points to $\Sigma_{n}$ to stabilize $\Sigma_{n}$, and we use the unique hyperbolic metric on $\Sigma_{n}$ to decompose $\Sigma_{n}$ into $\varepsilon$-thick part $\Sigma_{n}^{\varepsilon \text {-thick and }}$ 
$\varepsilon$-thin part $\Sigma_{n}^{\varepsilon \text {-thin }}$ according to the injectivity radius, for $\varepsilon>0$. Take a subsequence of $\Sigma_{n}$, still called $\Sigma_{n}$, such that $\Sigma_{n}$ converges to a nodal surface $\Sigma_{\infty}$ in the Deligne-Mumford sense. By continuing to add marked points to $\Sigma_{n}$, if necessary, one can keep track of all the sphere bubbles of $w_{n}$ as $n \rightarrow \infty$. Eventually, for fixed $\varepsilon>0$, we achieve that $\left.w_{n}\right|_{\Sigma_{n}^{\varepsilon-t h i c k}}$ has a uniformly gradient bound. By Ascoli-Arzela and elliptic estimates, we can extract a convergent subsequence of $w_{n}$, still called $w_{n}$. Now letting $\varepsilon$ tend to 0 and picking a diagonal subsequence, we get a convergent subsequence of $w_{n}$, still called $w_{n}$, with the limit $\left(\Sigma_{\infty}, w_{\infty} \mid \Sigma_{\infty}\right)$. By Theorem 4.2, we know that, around a puncture, the limit $\left.w_{\infty}\right|_{\Sigma_{\infty}}$ either has a removable singularity or converges to a Reeb orbit. But at the current stage, $w_{\infty}$ may not be defined around the nodal points.

Secondly, for $\varepsilon$ sufficiently small, the $\varepsilon$-thin part is a disjoint union of finite cylinders or half-finite cylinders. If $E_{\omega}\left(\left.w_{n}\right|_{\Sigma_{n}^{\varepsilon} \text {-thin }}\right) \rightarrow 0$ as $n \rightarrow \infty$, then the behavior of $\left.w_{n}\right|_{\Sigma_{n}^{\varepsilon} \text {-thin }}$ is controlled by Theorem 3.22. In this case, the convergence of $w_{n}$ in the thick part can be continuously extended over $\Sigma_{n}$. Otherwise, $\left.w_{n}\right|_{\Sigma_{n}^{\varepsilon} \text {-thin }}$ can have the additional broken trajectory degeneration. By adding more marked points to keep track of all of the broken trajectory, one has that $E_{\omega}\left(\left.w_{n}\right|_{\Sigma_{n}^{\varepsilon-\text { thin }}}\right) \rightarrow 0$ as $n \rightarrow \infty$.

\section{Acknowledgements}

Firstly, I would like to express my deepest gratitude to my advisor Yong-Geun Oh for suggesting such an interesting project. Secondly, I would like to thank Conan Leung for very patiently answering my questions about symplectic geometry. Thirdly, I would like to thank Lino Amorim, Garrett Alston, Dongning Wang, Rui Wang and Ke Zhu for all the fruitful discussions and valuable suggestions. Last but not least, I thank the anonymous referee for all the critiques and suggestions, which largely improved the quality of this paper.

\section{References}

[Audin and Lafontaine 1994] M. Audin and J. Lafontaine (editors), Holomorphic curves in symplectic geometry, Progress in Mathematics 117, Birkhäuser, Basel, 1994. MR 95i:58005 Zbl 0802.53001

[Bao 2014] E. Bao, "On Hofer energy for $J$-holomorphic curves for asymptotically cylindrical $J$ ", 2014. To appear in J. Symplectic Geom. arXiv 1303.4430

[Bourgeois 2002] F. Bourgeois, A Morse-Bott approach to contact homology, Ph.D. thesis, Stanford University, 2002, available at http://search.proquest.com/docview/305591502. MR 2703292 Zbl 1046.57017

[Bourgeois et al. 2003] F. Bourgeois, Y. Eliashberg, H. Hofer, K. Wysocki, and E. Zehnder, "Compactness results in symplectic field theory", Geom. Topol. 7 (2003), 799-888. MR 2004m:53152 Zbl 1131.53312

[Eliashberg 2007] Y. Eliashberg, "Symplectic field theory and its applications", pp. 217-246 in International Congress of Mathematicians, Vol. I, edited by M. Sanz-Solé et al., Eur. Math. Soc., Zürich, 2007. MR 2008f:53119 Zbl 1128.53059 
[Eliashberg et al. 2000] Y. Eliashberg, A. Givental, and H. Hofer, "Introduction to symplectic field theory", Geom. Funct. Anal. Special Volume, Part II (2000), 560-673. MR 2002e:53136 Zbl 0989.81114

[Gromov 1985] M. Gromov, "Pseudo holomorphic curves in symplectic manifolds", Invent. Math. 82:2 (1985), 308-347. Zbl 0592.53025

[Hofer 1993] H. Hofer, "Pseudoholomorphic curves in symplectizations with applications to the Weinstein conjecture in dimension three", Invent. Math. 114:3 (1993), 515-563. MR 94j:58064 Zbl 0797.58023

[Hofer and Viterbo 1992] H. Hofer and C. Viterbo, "The Weinstein conjecture in the presence of holomorphic spheres", Comm. Pure Appl. Math. 45:5 (1992), 583-622. MR 93h:58055 Zbl 0773.58021

[Hofer et al. 2001] H. Hofer, K. Wysocki, and E. Zehnder, "The asymptotic behavior of a finite energy plane”, preprint, 2001, available at http://e-collection.library.ethz.ch/eserv/eth:25385/eth25385-01.pdf.

[Hofer et al. 2002] H. Hofer, K. Wysocki, and E. Zehnder, "Finite energy cylinders of small area", Ergodic Theory Dynam. Systems 22:5 (2002), 1451-1486. MR 2003k:53121 Zbl 1041.57009

[Hummel 1997] C. Hummel, Gromov's compactness theorem for pseudo-holomorphic curves, Progress in Mathematics 151, Birkhäuser, Basel, 1997. MR 98k:58032 Zbl 0870.53002

Received January 19, 2014. Revised April 21, 2015.

ERKAO BAO

DEPARTMENT OF MATHEMATICS

UNIVERSITY OF CALIFORNIA, LOS ANGELES

520 Portola Plaza, Math Science Building, UClA

Los Angeles, CA 90095

UNITED STATES

bao@math.ucla.edu 


\title{
PACIFIC JOURNAL OF MATHEMATICS
}

\author{
msp.org/pjm
}

Founded in 1951 by E. F. Beckenbach (1906-1982) and F. Wolf (1904-1989)

\section{EDITORS}

Don Blasius (Managing Editor)

Department of Mathematics

University of California

Los Angeles, CA 90095-1555

blasius@math.ucla.edu

\author{
Paul Balmer \\ Department of Mathematics \\ University of California \\ Los Angeles, CA 90095-1555 \\ balmer@math.ucla.edu \\ Robert Finn \\ Department of Mathematics \\ Stanford University \\ Stanford, CA 94305-2125 \\ finn@math.stanford.edu \\ Sorin Popa \\ Department of Mathematics \\ University of California \\ Los Angeles, CA 90095-1555 \\ popa@math.ucla.edu
}

\author{
Vyjayanthi Chari \\ Department of Mathematics \\ University of California \\ Riverside, CA 92521-0135 \\ chari@math.ucr.edu \\ Kefeng Liu \\ Department of Mathematics \\ University of California \\ Los Angeles, CA 90095-1555 \\ liu@math.ucla.edu \\ Jie Qing \\ Department of Mathematics \\ University of California \\ Santa Cruz, CA 95064 \\ qing@ cats.ucsc.edu
}

\section{PRODUCTION}

Silvio Levy, Scientific Editor, production@msp.org

\section{SUPPORTING INSTITUTIONS}

ACADEMIA SINICA, TAIPEI

CALIFORNIA INST. OF TECHNOLOGY

INST. DE MATEMÁTICA PURA E APLICADA

KEIO UNIVERSITY

MATH. SCIENCES RESEARCH INSTITUTE

NEW MEXICO STATE UNIV.

OREGON STATE UNIV.

\author{
STANFORD UNIVERSITY \\ UNIV. OF BRITISH COLUMBIA \\ UNIV. OF CALIFORNIA, BERKELEY \\ UNIV. OF CALIFORNIA, DAVIS \\ UNIV. OF CALIFORNIA, LOS ANGELES \\ UNIV. OF CALIFORNIA, RIVERSIDE \\ UNIV. OF CALIFORNIA, SAN DIEGO \\ UNIV. OF CALIF., SANTA BARBARA
}

\author{
Daryl Cooper \\ Department of Mathematics \\ University of California \\ Santa Barbara, CA 93106-3080 \\ cooper@math.ucsb.edu \\ Jiang-Hua Lu \\ Department of Mathematics \\ The University of Hong Kong \\ Pokfulam Rd., Hong Kong \\ jhlu@maths.hku.hk \\ Paul Yang \\ Department of Mathematics \\ Princeton University \\ Princeton NJ 08544-1000 \\ yang@math.princeton.edu
}

These supporting institutions contribute to the cost of publication of this Journal, but they are not owners or publishers and have no responsibility for its contents or policies.

See inside back cover or msp.org/pjm for submission instructions.

The subscription price for 2015 is US \$420/year for the electronic version, and \$570/year for print and electronic.

Subscriptions, requests for back issues and changes of subscribers address should be sent to Pacific Journal of Mathematics, P.O. Box 4163, Berkeley, CA 94704-0163, U.S.A. The Pacific Journal of Mathematics is indexed by Mathematical Reviews, Zentralblatt MATH, PASCAL CNRS Index, Referativnyi Zhurnal, Current Mathematical Publications and Web of Knowledge (Science Citation Index).

The Pacific Journal of Mathematics (ISSN 0030-8730) at the University of California, c/o Department of Mathematics, 798 Evans Hall \#3840, Berkeley, CA 94720-3840, is published twelve times a year. Periodical rate postage paid at Berkeley, CA 94704, and additional mailing offices. POSTMASTER: send address changes to Pacific Journal of Mathematics, P.O. Box 4163, Berkeley, CA 94704-0163.

PJM peer review and production are managed by EditFLOW ${ }^{\circledR}$ from Mathematical Sciences Publishers.

\section{PUBLISHED BY}

\section{mathematical sciences publishers \\ nonprofit scientific publishing}

http://msp.org/

(C) 2015 Mathematical Sciences Publishers 


\section{PACIFIC JOURNAL OF MATHEMATICS}

Volume $278 \quad$ No. $2 \quad$ December 2015

Differential Harnack and logarithmic Sobolev inequalities along

Ricci-harmonic map flow

ABIMBOLA ABOLARINWA

On $J$-holomorphic curves in almost complex manifolds with

291

asymptotically cylindrical ends

ERKAO BAO

Integration of coupling Dirac structures

OLIVIER BRAHIC and RUI LOJA FERNANDES

Asymptotic behavior of Palais-Smale sequences associated with fractional Yamabe-type equations

Yi FANG and MARÍA DEL MAR GONZÁLEZ

$K$-theory and homotopies of 2-cocycles on higher-rank graphs

ELIZABETH GILLASPY

Fusion products and toroidal algebras

DENIZ KUS and PETER LiTTELMANN

Differential Harnack estimates for positive solutions to heat equation under Finsler-Ricci flow

\section{SAJJAD LAKZIAN}

On the one-endedness of graphs of groups

NichOLAS TOUIKAN

On the structure of vertex cuts separating the ends of a graph

GARETH R. WILKES 